\title{
EXISTENCE AND COMPUTATION OF PERIODIC TRAVELLING-WAVE SOLUTIONS OF A DISPERSIVE SYSTEM*
}

\author{
JUAN CARLOS MUÑOZ GRAJALES ${ }^{\dagger}$
}

\begin{abstract}
We establish existence of periodic travelling-wave solutions to a generalized Boussinesq system by using the topological degree theory for positive operators defined on a cone in an appropriate Banach space. Furthermore, we derive a high-accuracy pseudospectral solver based on a Fourier decomposition to construct numerical approximations of these stationary solutions. The numerical simulations are in perfect agreement with the theoretical results and new travelling-wave solutions of the system are computed which do not belong to the family of solutions proved to exist.
\end{abstract}

Key words. Boussinesq system, periodic travelling-wave solutions, pseudospectral methods.

AMS subject classifications. 42A45, 47A75, 35Q51, 76M45.

\section{Introduction}

Travelling waves exist as a consequence of a balance between nonlinear and dispersive effects present in a system, and they have played an important role in the last decades in the study of dynamics of wave propagation in a broad set of applications such as fluid dynamics, optics, acoustics, oceanography, and weather forecasting, among others. Thus to determine existence and properties of such type of solutions is a fundamental problem in the theory of ordinary and partial differential equations of great interest for both pure and applied mathematicians. Famous examples of partial differential equations which possess travelling-wave solutions include the Korteweg-de Vries equation (KdV) [17], the Benjamin-Ono model [2], the Benjamin-Bona-Mahony equation [1], and the Benney-Luke equation [4].

In analogy with the study performed for the set of equations mentioned above, the first purpose of the present paper is to discuss existence of even $2 l$-periodic travellingwave solutions in the form

$$
\eta(x, t)=\sum_{n} \eta_{n} e^{i(n \pi / l)(x-c t)}, \quad u(x, t)=\sum_{n} u_{n} e^{i(n \pi / l)(x-c t)}
$$

of the generalized Boussinesq system

$$
\begin{aligned}
& \eta_{t}+\delta \eta_{x x t}+\mu u_{x x t}+u_{x}+\theta u_{x x x}+\alpha\left(u^{p} \eta\right)_{x}=0, \\
& u_{t}+\delta u_{x x t}+\mu \eta_{x x t}+\eta_{x}+\theta \eta_{x x x}+\alpha\left(\frac{u^{p+1}}{p+1}\right)_{x}=0,
\end{aligned}
$$

where $\delta, \mu, \alpha, \theta$ are constants and $p \geq 1$ is an integer. For $p=1$ and $\mu=0$ this system is a model for the two-way propagation of water waves on the surface of a channel with flat bottom, considering the effects of weak dispersion and nonlinearity [5]. In the case $\mu<0, p=1$, Equation (1.1) is a variant of a system of two coupled KdV-type equations derived originally by J. A. Gear and R. Grimshaw [14] as a model to describe the strong interaction of weakly nonlinear, long waves. It is also worthwhile to mention the works by J. Morais [20] and V. Bisognin [7] on water wave models with dispersive terms

*Received: March 30, 2011; accepted (in revised version): November 29, 2011. Communicated by Paul Milewski.

${ }^{\dagger}$ Universidad del Valle, Departamento de Matemáticas, A.A. 25360, Cali, Colombia (jcarlmz@ yahoo.com). 
similar to those in System (1.1). Thus Boussinesq-type systems such as Equation (1.1) have been used in the study of wave problems in fluid mechanics. One of the main features that makes System (1.1) very interesting from the physical point of view is that a well known water wave model such as the generalized KdV equation emerges from this Boussinesq formulation. In fact, formally it can be shown (see Section 2) that if $\eta=\eta(x-c t), u=u(x-c t)$ is a solitary-wave solution (i.e. a travelling-wave solution which decays to zero at infinity) of System (1.1) with speed $c>0$ then $N(x, t)=$ $\eta\left(x-c^{2} t\right), U(x, t)=u\left(x-c^{2} t\right)$ satisfy up to order two in the parameters $\alpha, \theta, \mu, \delta$, the generalized $\mathrm{KdV}$ equations

$$
\begin{aligned}
& U_{t}+U_{x}+\gamma_{1} U^{p} U_{x}+\gamma_{2} U_{x x x}=0, \\
& N_{t}+N_{x}+\frac{\gamma_{1}}{c^{p}} N^{p} N_{x}+\gamma_{2} N_{x x x}=0,
\end{aligned}
$$

where

$$
\gamma_{1}=\alpha c(p+2), \quad \gamma_{2}=-2\left(\delta c^{2}+\mu c-\theta\right) .
$$

It is important to point out that except in the simplest examples, such as the KdV equation for which travelling-wave solutions are known explicitly, for a general dispersive system such as (1.1) (with $p>1$ ), closed-form solutions are unknown, and thus the existence issue and computation of them are nontrivial problems which must be studied through both analytical and numerical means. We also mention that travelling-wave solutions of these systems are of importance in the study of river bores [8]. An example of a similar system used in the study of internal waves is given in $[25]$.

In the present paper, we explore existence of periodic travelling-wave solutions to System (1.1) using ideas from Benjamin et al [3] in the framework of solitary wave solutions of some scalar dispersive equations, $\mathrm{H}$. Chen [10] in the case of periodic travelling-wave solutions of a scalar dispersive equation, and from $\mathrm{H}$. Chen et al [11] who study periodic travelling-waves of a Boussinesq system with quadratic nonlinear terms. In the present periodic setting, we apply the positive operator theory introduced by Krasnosel'skii [18, 19] to the problem formulated in the Fourier transformed variables, rather than in the original physical variables, which allows us to obtain a theory with fewer hypotheses than in the work by Benjamin et al [3]. Remark that $\mathrm{H}$. Chen developed in [10] the main breakthrough in the application of this method to dispersive-type equations in a periodic domain. We also point out that this topological approach is found to be free from restrictions on wave amplitude (controlled in this case by the parameter $\alpha$ ) and only depends on the parameters appearing in the linear dispersive terms of System (1.1). Our results show existence of even travelling-wave solutions with period $2 l$ of this Boussinesq formulation provided that the half-period $l$ is large enough and the wave velocity $c$ is within the range

$$
\frac{\theta}{\mu}>c>\max \left\{1, \frac{\theta}{\delta+\mu},-\frac{\mu+\sqrt{\mu^{2}+4 \delta \theta}}{2 \delta}\right\},
$$

with $\theta<0, \delta<\mu<0$. Notice that this range does not depend on the exponent $p$ present in the nonlinear terms of Equation (1.1).

As mentioned above, nonconstant exact stationary solutions of System (1.1) are not available for $p>1$. The second purpose of this paper is to construct approximations to periodic even travelling-wave solutions of System (1.1) whose existence is 
guaranteed by the theoretical results. This is performed by using a new numerical scheme that features a pseudospectral method with the Fourier basis to approximate the spatial structure and a Newton's iteration for solving the system of nonlinear equations generated. In addition to this family of solutions, we discover new periodic noneven solutions computed through this numerical tool. We are aware of very few works where this type of solutions to Boussinesq-type systems for water waves are computed. From the theoretical point of view, the effect of the exponent $p$ on the behavior of solutions of System (1.1) is addressed in [24] but there is still a lack of theory for determining the way that the geometric shape of travelling-wave solutions of System (1.1) is influenced by the parameter $p$. Regarding numerical solutions to one-dimensional Boussinesq-type systems, we mention the work by Chen [9] which introduces a shooting-type method for approximating solitary-wave solutions to a Boussinesq-type system with quadratic nonlinear terms. In general, this technique becomes computationally expensive since two systems of ordinary differential equations must be solved at each step of the corresponding Newton's iteration. We remark that in our work, only one linear system must be solved in each Newton's iteration in order to approximate the zero of the vector field involved. Thus, the solver proposed becomes inexpensive, computationally efficient, and convergent in a few iterations in all experiments performed. This numerical scheme allows us to visualize the shape of periodic solutions to System (1.1) (for different values of the model's parameters and wave speed) and validate the mathematical theory developed in this paper.

In the work by Chen et al [11] a family of periodic travelling-wave solutions are found for the same Boussinesq formulation as in [9] by using the Jacobi elliptic function series, and Bona and Chen proposed in [6] a numerical scheme for solving a regularized Boussinesq formulation with symmetric dispersive terms of third order by applying a finite-difference strategy. Other papers related to numerical computations of solutions to one-dimensional Boussinesq systems are [21, 22, 23].

The paper is organized as follows: In Section 2, we discuss the derivation of Equation (1.1) and the KdV-type Equation (1.2). In Section 3, we introduce notation and results necessary in order to develop existence theory of travelling-wave solutions of System (1.1). In Section 4, we reformulate the problem as one of finding a fixed point of a nonlinear positive operator defined on a cone in an appropriate Banach space. In Section 5, we establish existence of a family of travelling-wave solutions of System (1.1) parametrized by the wave speed $c$ within the range given in (1.3), provided that the half-period $l$ is large enough. Finally in Section 6, we introduce the numerical solver employed to compute periodic travelling-wave solutions of System (1.1) and illustrate the theoretical results.

\section{Derivation of the Boussinesq system}

As mentioned above, system (1.1) with $p=1$ is motivated by the work by J. A. Gear and R. Grimshaw [14, p. 250] who derived a system of two coupled KdV-type equations in the form

$$
\begin{aligned}
& u_{t}+\alpha_{1} u_{x x x}+\alpha_{2} v_{x x x}+\alpha_{3} u u_{x}+\alpha_{4} v v_{x}+\alpha_{5}(u v)_{x}=0, \\
& v_{t}+\beta_{1} v_{x x x}+\beta_{2} u_{x x x}+\beta_{3} v v_{x}+\beta_{4} u u_{x}+\beta_{5}(u v)_{x}+\beta_{6} v_{x}=0 .
\end{aligned}
$$

Here $\alpha_{i}, \beta_{i}$ are real constants. We consider the case $\alpha_{3}=\alpha_{4}=1, \alpha_{5}=0, \beta_{3}=\beta_{4}=\beta_{6}=$ $0, \beta_{5}=1$. Let $\alpha>0$ be a small parameter and further suppose that $\alpha_{1}, \alpha_{2}, \beta_{1}, \beta_{2}$ are of order $O(\alpha)$. Now making the change of variables

$$
u=1+\alpha U, \quad v=1+\alpha \eta,
$$


equations (2.1)-(2.2) transform into

$$
\begin{aligned}
& U_{t}+\alpha_{1} U_{x x x}+\alpha_{2} \eta_{x x x}+U_{x}+\alpha U U_{x}+\eta_{x}+\alpha \eta \eta_{x}=0, \\
& \eta_{t}+\beta_{1} \eta_{x x x}+\beta_{2} U_{x x x}+U_{x}+\eta_{x}+\alpha(U \eta)_{x}=0 .
\end{aligned}
$$

By introducing the variable

$$
X=x-t
$$

we obtain the system

$$
\begin{aligned}
& U_{t}+\alpha_{1} U_{X X X}+\alpha_{2} \eta_{X X X}+\eta_{X}+\alpha U U_{X}+\alpha \eta \eta_{X}=0 \\
& \eta_{t}+\beta_{1} \eta_{X X X}+\beta_{2} U_{X X X}+U_{X}+\alpha(U \eta)_{X}=0 .
\end{aligned}
$$

The equations above yield the following leading order approximations:

$$
\eta_{t}=-U_{X}, \quad U_{t}=-\eta_{X}
$$

Let $\theta$ a real constant of order $O(\alpha)$. Using (2.5), equations (2.3)-(2.4) lead to

$$
\begin{aligned}
& U_{t}-\alpha_{1} \eta_{X X t}+\left(\theta-\alpha_{2}\right) U_{X X t}+\theta \eta_{X X X}+\alpha U U_{X}+\eta_{X}+\alpha \eta \eta_{X}=0, \\
& \eta_{t}-\beta_{1} U_{X X t}+\left(\theta-\beta_{2}\right) \eta_{X X t}+\theta U_{X X X}+U_{X}+\alpha(U \eta)_{X}=0 .
\end{aligned}
$$

Therefore in this paper we consider a version of System (2.6)-(2.7) where $\delta=\theta-$ $\beta_{2}=\theta-\alpha_{2}, \mu=-\beta_{1}=-\alpha_{1}$ and the nonlinear term $\alpha \eta \eta_{X}$ in Equation (2.6) has been neglected. This is just a convenient mathematical simplification in order to reduce the number of parameters and nonlinear terms involved in the analysis. Furthermore, we point out that other Boussinesq formulations could be derived by altering the form of dispersive terms in equations (2.6)-(2.7) with the help of relationships (2.5).

An interesting feature in regard to System (1.1) is that it is closely related to the generalized $\mathrm{KdV}$ equation. Suppose that $u(x, t)=u(x-c t), \eta(x, t)=\eta(x-c t)$ is a solitary wave solution with speed $c$ of System (1.1). Then the pair $(u, \eta)$ must satisfy the system of ordinary differential equations:

$$
\begin{aligned}
& -c \eta+u+(\theta-\mu c) u^{\prime \prime}-\delta c \eta^{\prime \prime}=-u^{p} \eta \\
& \eta-c u-\delta c u^{\prime \prime}+(\theta-\mu c) \eta^{\prime \prime}=-\frac{u^{p+1}}{p+1}
\end{aligned}
$$

Observe that system above can be rewritten as

$$
\alpha\left(u^{p} \eta\right)=B \eta-A u, \quad \frac{\alpha u^{p+1}}{p+1}=B u-A \eta,
$$

where $A$ and $B$ are second-order linear operators defined as $A=I-(\mu c-\theta) \partial_{x}^{2}$ and $B=c\left(I+\delta \partial_{x}^{2}\right)$. Applying the operators $A$ and $B$ to the previous equations respectively yields

$$
\alpha A\left(u^{p} \eta\right)=A B \eta-A^{2} u, \quad \alpha B \frac{\alpha u^{p+1}}{p+1}=B^{2} u-B A \eta .
$$

Adding the equations above we obtain that

$$
\alpha\left(A\left(u^{p} \eta\right)+B \frac{u^{p+1}}{p+1}\right)=\left(B^{2}-A^{2}\right) u
$$


We point out the following approximations:

$$
\left.B^{2}=c^{2}\left(I+2 \delta \partial_{x}^{2}\right)+O\left(\delta^{2}\right), \quad A^{2}=I-2(\mu c-\theta) \delta \partial_{x}^{2}\right)+O\left(\mu^{2}, \theta^{2}, \mu \theta\right) .
$$

Since $\alpha B u-\alpha A \eta=\frac{\alpha^{2} u^{p+1}}{p+1}=O\left(\alpha^{2}\right)$, we deduce that

$$
\begin{gathered}
\alpha c\left(I+\delta \partial_{x}^{2}\right) u-\alpha\left(I-(\mu c-\theta) \partial_{x}^{2}\right) \eta=O\left(\alpha^{2}\right), \\
\alpha c u-\alpha \eta=O(\alpha \delta, \alpha \mu, \alpha \theta),
\end{gathered}
$$

from which follows that

$$
\eta=c u+O(\alpha, \theta, \mu, \delta) .
$$

Observe also that

$$
\begin{aligned}
& \alpha A=\alpha I-\alpha(\mu c-\theta) \partial_{x}^{2}=\alpha I+O(\alpha \mu, \alpha \theta), \\
& \alpha B=\alpha c\left(I+\delta \partial_{x}^{2}\right)=\alpha c I+\alpha c \delta \partial_{x}^{2}=\alpha c I+O(\alpha \delta) .
\end{aligned}
$$

Using these facts and substituting the approximations for the operators $A$ and $B$ into Equation (2.8), we reach that

$$
\alpha u^{p} \eta+\frac{\alpha c u^{p+1}}{p+1}=\left(c^{2}-1\right) u+2\left(\delta c^{2}+(\mu c-\theta)\right) u^{\prime \prime}+\text { second-order terms in } \alpha, \theta, \mu, \delta .
$$

We get, after neglecting second-order terms in $\alpha, \delta, \theta, \mu$ and using Equation (2.9),

$$
\alpha c\left(\frac{p+2}{p+1}\right) u^{p+1}+\left(1-c^{2}\right) u-2\left(\delta c^{2}+\mu c-\theta\right) u^{\prime \prime}=0 .
$$

Therefore $U(x, t)=u\left(x-c^{2} t\right.$ ) is a solution (except for quadratic terms in $\alpha, \mu, \delta, \theta$ ) of the generalized $\mathrm{KdV}$ equation

$$
U_{t}+U_{x}+\gamma_{1} U^{p} U_{x}+\gamma_{2} U_{x x x}=0
$$

where

$$
\gamma_{1}=\alpha c(p+2), \quad \gamma_{2}=-2\left(\delta c^{2}+\mu c-\theta\right) .
$$

Analogously $N(x, t)=\eta\left(x-c^{2} t\right)$ is a solution (except for quadratic terms in $\left.\alpha, \mu, \delta, \theta\right)$ of

$$
N_{t}+N_{x}+\frac{\gamma_{1}}{c^{p}} N^{p} N_{x}+\gamma_{2} N_{x x x}=0
$$

\section{Notation and auxiliary results}

In this section, we recall definitions and notations employed in this work. Let $\mathbb{C}$ denote the complex field, $1 \leq p<\infty$, and $l_{p}$ the Banach space

$$
l_{p}:=\left\{\mathbf{u}=\left\{u_{n}\right\}_{n=-\infty}^{\infty}: u_{n} \in \mathbb{C}, \sum_{n=-\infty}^{\infty}\left|u_{n}\right|^{p}<\infty\right\},
$$




$$
\|\mathbf{u}\|_{p}^{p}:=\sum_{n=-\infty}^{\infty}\left|u_{n}\right|^{p}
$$

For $p=\infty$, we define the Banach space

$$
l_{\infty}:=\left\{\mathbf{u}=\left\{u_{n}\right\}_{n=-\infty}^{\infty}: u_{n} \in \mathbb{C}, \sup _{-\infty<n<\infty}\left|u_{n}\right|<\infty\right\}
$$

with its usual norm

$$
\|\mathbf{u}\|_{\infty}:=\sup _{-\infty<n<\infty}\left|u_{n}\right| .
$$

Recall that any sequence $\mathbf{f}=\left\{f_{n}\right\}_{n} \in l_{2}$ defines a periodic function $f$ of period $2 l$, where

$$
f(x)=\sum_{n=-\infty}^{\infty} f_{n} e^{\frac{i n \pi x}{l}}
$$

It is also worth noting that for any $1 \leq p<q<\infty, l_{p} \subset l_{q}$, and for any $\mathbf{u}=\left\{u_{n}\right\} \in$ $l_{p},\|\mathbf{u}\|_{q} \leq\|\mathbf{u}\|_{p}$. Furthermore, if $1 \leq r_{1}, \ldots r_{N} \leq \infty$ satisfy $\frac{1}{r_{1}}+\ldots . \frac{1}{r_{N}}=N-1$ and $\mathbf{u}^{1}=$ $\left\{u_{n}^{(1)}\right\} \in l_{r_{1}}, \ldots, \mathbf{u}^{N}=\left\{u_{n}^{(N)}\right\} \in l_{r_{N}}$ then

$$
\mathbf{w}=\left\{w_{n}\right\}=\mathbf{u}^{1} * \ldots * \mathbf{u}^{N} \in l_{\infty},
$$

where $w_{n}=\sum_{k_{1}+\ldots+k_{N}=n} u_{k_{1}}^{(1)} \ldots u_{k_{N}}^{(N)}$, and

$$
\|\mathbf{w}\|_{\infty} \leq\left\|\mathbf{u}^{(1)}\right\|_{r_{1} \ldots} \ldots \mathbf{u}^{(N)} \|_{r_{N}} .
$$

Now we include a brief review of some results from the functional analysis of positive operators following the paper of Benjamin et al [3]. Other references on this theme are $[18,19]$ and $[13]$. Let $X$ be a Banach space equipped with the norm \|\|$_{X}$. We say that a closed subset $K \subset X$ is a cone, if the following conditions are satisfied:

1. $\lambda K \equiv\{\lambda f: f \in K\} \subset K$ for all $\lambda \geq 0$.

2. $K+K \equiv\{f+g: f, g \in K\} \subset K$,

3. $K \cap\{-K\} \equiv K \cap\{-f: f \in K\}=\{0\}$.

For any $0<r<R<\infty$, let us denote

$$
\begin{aligned}
& B_{r}=\left\{f \in X:\|f\|_{X}<r\right\}, \quad \partial B_{r}=\left\{f \in X:\|f\|_{X}=r\right\}, \\
& K_{r}=K \cap B_{r}, \quad \partial K_{r}=K \cap \partial B_{r}, \\
& K_{r}^{R}=\left\{f \in K: r<\|f\|_{X}<R\right\} .
\end{aligned}
$$

A operator $\mathbf{A}$ defined on $K$ is called positive if $\mathbf{A}(K) \subset K$. A triple $(K, \mathbf{A}, U)$ is said to be admissible if

1. $K$ is a convex subset of $X$,

2. $U \subset K$ is open in the relative topology on $K$,

3. $\mathbf{A}: K \rightarrow K$ is continuous and $\mathbf{A}(U)$ is a subset of a compact set in $K$ and 
4. A has no fixed points on $\partial U$, the boundary of the open set $U$ in the relative topology on $K$.

If $(K, \mathbf{A}, U)$ is admissible and $\mathbf{A}$ is a constant function on $K$ (i.e., there exists some $a \in K$ such that $\mathbf{A} x=a$ for all $x \in K$ ), we define the fixed point index of the positive operator $\mathbf{A}$ on $U$ as

$$
i(K, \mathbf{A}, U):=\left\{\begin{array}{lll}
1 & \text { if } & a \in U, \\
0 & \text { if } & a \notin U .
\end{array}\right.
$$

An important result with regard to this concept is the fact that if $(K, \mathbf{A}, U)$ is admissible and $i(K, \mathbf{A}, U) \neq 0$, then $A$ has at least one fixed point in $U$. Furthermore, we have the following lemmas which will be useful in developing the theory in the current research. The operator $\mathbf{A}$ is positive, continuous, and compact on the cone $K$. We refer the reader to the work by Benjamin et al [3] for details.

Lemma 3.1. Suppose that $0<\rho<\infty$ and that either

(a) $\boldsymbol{A} x-x \notin K$ for all $x \in \partial K_{\rho}$, or

(b) $t \boldsymbol{A} x \neq x$ for all $x \in \partial K_{\rho}$ and all $t \in[0,1]$.

Then $i\left(K, \boldsymbol{A}, K_{\rho}\right)=1$.

Lemma 3.2. Suppose that $0<\rho<\infty$ and that either

(a) $x-\boldsymbol{A} x \notin K$ for all $x \in \partial K_{\rho}$, or $\lambda \geq 0$.

$(\tilde{b})$ there exists a non-zero $\tilde{x} \in K$ such that $x-\boldsymbol{A} x \neq \lambda \tilde{x}$ for all $x \in \partial K_{\rho}$ and all

Then $i\left(K, \boldsymbol{A}, K_{\rho}\right)=0$.

Lemma 3.3. Let $(K, \boldsymbol{A}, U)$ be admissible. If there exists a non-zero $\tilde{x} \in K$ such that $x-\boldsymbol{A} x \neq \lambda \tilde{x}$ for all $x \in \partial U$ and all $\lambda \geq 0$, then $i(K, \boldsymbol{A}, U)=0$.

As a consequence of the first two lemmas we have the following theorem:

TheOREm 3.4. Suppose that either (a) or (b) holds for an $r$ satisfying $0<r<\infty$ and that either $(\tilde{a})$ or $(\tilde{b})$ holds for an $R$ satisfying $r<R<\infty$. Then $A$ has at least one fixed point in $K_{r}^{R} \equiv\left\{f \in K, r<\|f\|_{X}<R\right\}$. Moreover, $i\left(K, \boldsymbol{A}, K_{r}^{R}\right)=-1$.

\section{Problem setting}

We consider the dispersive Boussinesq system

$$
\begin{aligned}
& \eta_{t}+\delta \eta_{x x t}+\mu u_{x x t}+u_{x}+\theta u_{x x x}+\alpha\left(u^{p} \eta\right)_{x}=0, \\
& u_{t}+\delta u_{x x t}+\mu \eta_{x x t}+\eta_{x}+\theta \eta_{x x x}+\alpha\left(\frac{u^{p+1}}{p+1}\right)_{x}=0
\end{aligned}
$$

where $\delta, \mu, \theta$ are real constants with some restrictions which will be established later. The parameter $p$ is an integer greater or equal to 1 . For simplicity we take $\alpha=1$ since the analysis does not depend on this parameter. In this work, we are interested in establishing if the system above has periodic travelling-wave solutions of the form

$$
\eta(x, t)=\eta_{c}(x-c t), \quad u(x, t)=u_{c}(x-c t),
$$

where $\eta_{c}$ and $u_{c}$ are periodic functions and the parameter $c$ is the wave speed. For simplicity, we denote $\eta=\eta_{c}, u=u_{c}$. The equations for travelling-wave solutions of 
System (4.1) take the form:

$$
\begin{aligned}
& -c \eta^{\prime}+u^{\prime}+(\theta-\mu c) u^{\prime \prime \prime}-\delta c \eta^{\prime \prime \prime}=-\left(u^{p} \eta\right)^{\prime} \\
& \eta^{\prime}-c u^{\prime}-\delta c u^{\prime \prime \prime}+(\theta-\mu c) \eta^{\prime \prime \prime}=-\left(\frac{u^{p+1}}{p+1}\right)^{\prime}
\end{aligned}
$$

Integrating Equation (4.2) we obtain

$$
\begin{aligned}
& -c \eta+u+(\theta-\mu c) u^{\prime \prime}-\delta c \eta^{\prime \prime}=-u^{p} \eta \\
& \eta-c u-\delta c u^{\prime \prime}+(\theta-\mu c) \eta^{\prime \prime}=-\frac{u^{p+1}}{p+1}
\end{aligned}
$$

Here we will assume that the constants of integration are zero. It is important to note that the system above admits constant solutions. First of all, $(\eta, u)=(0,0)$ is a trivial solution. Other constant solution can be derived by solving the system

$$
-c p_{0}+q_{0}=-q_{0}^{p} p_{0}, \quad p_{0}-c q_{0}=-\frac{q_{0}^{p+1}}{p+1} .
$$

Eliminating the variable $p_{0}$ from the equations above yields

$$
q_{0}^{2 p}-c(p+2) q_{0}^{p}+(p+1)\left(c^{2}-1\right)=0,
$$

from where we obtain that

$$
q_{0}=\left(\frac{c(p+2)-\sqrt{p^{2} c^{2}+4 p+4}}{2}\right)^{1 / p}
$$

and $p_{0}$ can be obtained from the equation

$$
p_{0}=\frac{q_{0}}{c-q_{0}^{p}} .
$$

If $c>1$ then it is easy to see that $p_{0} \geq q_{0} \geq(c-1)^{1 / p}>0$. Thus $\eta=p_{0}, u=q_{0}$ is another constant solution of System (4.3). We are interested in finding non-constant solutions of the travelling-wave Equation (4.3). These solutions $\eta=\eta(\xi), u=u(\xi)$ with period $2 l$ can be expanded in Fourier series as

$$
\eta=\sum_{n} \eta_{n} e^{\frac{i n \pi}{l} \xi}, \quad u=\sum_{n} u_{n} e^{\frac{i n \pi}{l} \xi} .
$$

By substituting expressions (4.4) into Equation (4.3), it follows that

$$
\left(\begin{array}{l}
\eta_{n} \\
u_{n}
\end{array}\right)=D_{n}^{-1} \cdot\left(\begin{array}{c}
\left(\mathbf{u}^{p} \times \boldsymbol{\eta}\right)_{n} \\
\frac{1}{p+1}(\mathbf{u} \times \ldots \times \mathbf{u})_{n}
\end{array}\right)
$$

where

$$
D_{n}:=\left(\begin{array}{cc}
c\left(1-\frac{\delta n^{2} \pi^{2}}{l^{2}}\right) & (\theta-\mu c) \frac{n^{2} \pi^{2}}{l^{2}}-1 \\
(\theta-\mu c) \frac{n^{2} \pi^{2}}{l^{2}}-1 & c\left(1-\frac{\delta n^{2} \pi^{2}}{l^{2}}\right)
\end{array}\right),
$$

and $(\mathbf{u} \times \ldots \times \mathbf{u})_{n}$, and $\left(\mathbf{u}^{p} \times \boldsymbol{\eta}\right)_{n}$ are sequences defined by

$$
(\mathbf{u} \times \ldots \times \mathbf{u})_{n}:=\sum_{k_{1}+\ldots+k_{p+1}=n} u_{k_{1}} \ldots . u_{k_{p+1}}
$$




$$
\left(\mathbf{u}^{p} \times \boldsymbol{\eta}\right)_{n}:=\sum_{k_{1}+\ldots+k_{p+1}=n} u_{k_{1}} \ldots . u_{k_{p}} \cdot \eta_{k_{p+1}} .
$$

Note that

$$
D_{n}^{-1}:=\frac{1}{\operatorname{det} D_{n}}\left(\begin{array}{cc}
c\left(1-\frac{\delta n^{2} \pi^{2}}{l^{2}}\right) & 1-(\theta-\mu c) \frac{n^{2} \pi^{2}}{l^{2}} \\
1-(\theta-\mu c) \frac{n^{2} \pi^{2}}{l^{2}} & c\left(1-\frac{\delta n^{2} \pi^{2}}{l^{2}}\right)
\end{array}\right)
$$

with

$$
\operatorname{det} D_{n}=c^{2}-1+\left(2(\theta-\mu c)-2 \delta c^{2}\right) \frac{n^{2} \pi^{2}}{l^{2}}+\left(c^{2} \delta^{2}-(\theta-\mu c)^{2}\right) \frac{n^{4} \pi^{4}}{l^{4}}
$$

In order for the matrix $D_{n}$ to be invertible it is sufficient that $\operatorname{det} D_{n}$ be positive for all $n$. Thus, we reach the admissible region for the travelling-wave speed:

$$
\frac{\theta}{\mu}>c>\max \left\{1, \frac{\theta}{\delta+\mu},-\frac{\mu+\sqrt{\mu^{2}+4 \delta \theta}}{2 \delta}\right\}
$$

where $\theta<0, \delta<\mu<0$. Condition (4.8) results from requiring that

$$
\begin{aligned}
& c^{2}>1, \\
& 2(\theta-\mu c)-2 \delta c^{2}>0, \\
& c^{2} \delta^{2}-(\theta-\mu c)^{2}>0 .
\end{aligned}
$$

Thus we have that $\operatorname{det}\left(D_{n}\right)>0$. Recall that $\theta<0, \delta<\mu<0$. The first observation is that in [11] $\mu=0$, and the corresponding condition for $c$ is

$$
c^{2}>\max \left\{1, \frac{\theta^{2}}{\delta^{2}}\right\}
$$

which results directly from inequality (4.11). Observe that condition (4.10) is satisfied trivially in this case. On the other hand, when $\mu<0$, inequalities (4.9), (4.11) are satisfied when

$$
c>1, \quad \frac{\theta}{\mu}>c>\frac{\theta}{\delta+\mu} .
$$

In order to satisfy the remaining inequality (4.10), we must restrict the wave velocity $c$ so that the quadratic polynomial $f(c)=\theta-\mu c-\delta c^{2}$ be positive. For this purpose, since

$$
c^{*}=-\frac{\mu+\sqrt{\mu^{2}+4 \delta \theta}}{2 \delta}
$$

is the unique positive root of $f(c), f(0)=\theta$, and $\delta<0$, we conclude that if

$$
\frac{\theta}{\mu}>c>\max \left\{1, \frac{\theta}{\delta+\mu}, c^{*}\right\}
$$

then conditions (4.9)-(4.11) are satisfied. This explains why condition (4.8) can not be specialized to the case $\mu=0$. 
Consider the Banach space $X=l_{(p+1) / p} \times l_{(p+1) / p}$ and define the subset

$$
K:=\left\{\left(\eta_{n}, u_{n}\right) \in X:\left(\eta_{n}, u_{n}\right)=\left(\eta_{-n}, u_{-n}\right), \eta_{0} \geq \eta_{1} \geq \ldots \geq 0, u_{0} \geq u_{1} \geq \ldots \geq 0\right\} .
$$

The space $X$ is equipped with the norm

$$
\|(\boldsymbol{\eta}, \mathbf{u})\|^{(p+1) / p}=\left\|\left(\eta_{n}, u_{n}\right)\right\|^{(p+1) / p}:=\|\boldsymbol{\eta}\|_{(p+1) / p}^{(p+1) / p}+\|\mathbf{u}\|_{(p+1) / p}^{(p+1) / p} .
$$

We remark that due to $p \geq 1$ we have $X \subset l_{2} \times l_{2}$. It can also verified that the set $K$ is a cone. Now for a sequence $\mathbf{w}=(\boldsymbol{\eta}, \mathbf{u})=\left(\eta_{n}, u_{n}\right) \in X$, we define the nonlinear operator

$$
(\mathbf{A w})_{n}:=D_{n}^{-1}\left(\begin{array}{c}
\left(\mathbf{u}^{p} \times \boldsymbol{\eta}\right)_{n} \\
\frac{1}{p+1}(\mathbf{u} \times \ldots \times \mathbf{u})_{n}
\end{array}\right) .
$$

It is important to note that the problem of finding solutions of system (4.3) reduces to searching for fixed points of the operator $\mathbf{A}$ on an appropriate space. In the next section we will establish that the operator $\mathbf{A}$ has a fixed point in the cone $K$ which is different from $\mathbf{w}=\left(\mathbf{p}^{*}, \mathbf{q}^{*}\right) \in X$, with $\mathbf{p}^{*}=\left(0,0, \ldots, p_{0}, \ldots, 0\right), \mathbf{q}^{*}=\left(0,0, \ldots, q_{0}, 0, \ldots, 0\right)$, and the origin $\mathbf{w}=\mathbf{0}$ of the Banach space $X$.

\section{Main results}

In the following we will assume that the wave speed $c$ is located within the admissible region given in (4.8).

Lemma 5.1. The map $\boldsymbol{A}$ is a continuous, positive, and compact operator on the cone $K$.

Proof. For $\mathbf{w}=(\boldsymbol{\eta}, \mathbf{u}) \in K$, let be

$$
t_{n}=(\mathbf{u} \times \ldots \times \mathbf{u})_{n}=\sum_{k_{1}+\ldots .+k_{p+1}=n} u_{k_{1}} u_{k_{2}} \ldots . u_{k_{p+1}} .
$$

It can be shown that for $p=1$, we have $t_{n}=t_{-n}$. For a large number of terms we proceed by induction on $p$. Suppose that the result is true for $p$ and let us calculate

$$
\begin{aligned}
t_{-n} & =\sum_{k_{1}+\ldots+k_{p+1}=-n} u_{k_{1}} u_{k_{2}} \ldots u_{k_{p+1}} \\
& =\sum_{k_{p+1}=-\infty}^{\infty}\left(\sum_{k_{1}+\ldots .+k_{p}=-n-k_{p+1}} u_{k_{1}} u_{k_{2}} \ldots . u_{k_{p}}\right) u_{k_{p+1}} \\
& =\sum_{k_{p+1}=-\infty}^{\infty}\left(\sum_{k_{1}+\ldots .+k_{p}=n-k_{p+1}} u_{k_{1}} u_{k_{2}} \ldots . u_{k_{p}}\right) u_{k_{p+1}} \quad \text { (by induction hypothesis) } \\
& =\sum_{k_{1}+\ldots . k_{p+1}=n} u_{k_{1}} u_{k_{2} \ldots . . . u_{k_{p+1}}}=t_{n} .
\end{aligned}
$$

Then $t_{-n}=t_{n}$ for $p+1$. Moreover, for $p=1$ we have that $t_{n} \geq t_{n+1}$ and by using again a argument of induction this is established for $p>1$.

On the other hand,

$$
0 \leq t_{n}=\sum_{k_{1}+\ldots .+k_{p+1=n}} u_{k_{1}} u_{k_{2}} \ldots . . u_{k_{p}} u_{k_{p+1}} \leq\|u\|_{\frac{p+1}{p}}^{p+1}
$$




$$
0 \leq s_{n}=\sum_{k_{1}+\ldots .+k_{p+1=n}} u_{k_{1}} u_{k_{2}} \ldots u_{k_{p}} \eta_{k_{p+1}} \leq\|u\|_{\frac{p+1}{p}}^{p}\|\eta\|_{\frac{p+1}{p}}
$$

and

$$
\begin{aligned}
\left|D_{n}^{-1}(i, j)\right| & \leq \max _{1 \leq j \leq 2} \sum_{i=1}^{2}\left|D_{n}^{1}(i, j)\right|=\left\|D_{n}^{-1}\right\|_{\infty} \\
& =\frac{c\left(1-\frac{\delta n^{2} \pi^{2}}{l^{2}}\right)+1-(\theta-\mu c) \frac{n^{2} \pi^{2}}{l^{2}}}{c^{2}-1+\left(2(\theta-\mu c)-2 \delta c^{2}\right) \frac{n^{2} \pi^{2}}{l^{2}}+\left(c^{2} \delta^{2}-(\theta-\mu c)^{2}\right) \frac{n^{4} \pi}{l^{4}}} \\
& =\frac{c\left(1-\delta n^{2} \frac{\pi^{2}}{l^{2}}\right)+1-(\delta-\mu c) \frac{\mu^{2} \pi^{2}}{l^{2}}}{\left(c\left(1-\delta n^{2} \frac{\pi^{2}}{l^{2}}\right)+(\theta-\mu c) \frac{n^{2} \pi^{2}}{l^{2}}-1\right)\left(c\left(1-\frac{\delta n^{2} \pi^{2}}{l^{2}}\right)-(\theta-\mu c) \frac{n^{2} \pi^{2}}{l^{2}}+1\right)} \\
& =\frac{1}{c\left(1-\frac{\delta n^{2} \pi^{2}}{l^{2}}\right)+(\theta-\mu c) \frac{n^{2} \pi^{2}}{l^{2}}-1} .
\end{aligned}
$$

Furthermore the entries of the matrix $D_{n}^{-1}$ are decreasing in $|n|$ and

$$
\sum_{n}\left\|D_{n}^{-1}\right\|_{\infty}^{\frac{p+1}{p}}=\sum_{n}\left|\frac{1}{c-1+(\theta-\mu c-\delta c) \frac{n^{2} \pi^{2}}{l^{2}}}\right|^{\frac{p+1}{p}}<\infty,
$$

since $\frac{p+1}{p}>1$.

In the following we will denote for simplicity $\|\cdot\|=\|\cdot\|_{\frac{p+1}{p}}$. Suppose that $\mathbf{w} \in K$. From the results given above, it follows that

$$
(\mathbf{A w})_{n}=(\mathbf{A w})_{-n}, \quad(\mathbf{A w})_{n} \geq(\mathbf{A w})_{n+1},
$$

and

$$
\begin{aligned}
\|\mathbf{A} \mathbf{w}\|_{X}^{\frac{p+1}{p}}= & \left\|D_{n}(1,1)\left(\mathbf{u}^{p} \times \boldsymbol{\eta}\right)_{n}+\frac{D_{n}(1,2)}{p+1}(\mathbf{u} \times \ldots \times \mathbf{u})_{n}\right\|^{\frac{p+1}{p}} \\
& +\left\|D_{n}(2,1)\left(\mathbf{u}^{p} \times \boldsymbol{\eta}\right)_{n}+\frac{D_{n}(2,2)}{p+1}(\mathbf{u} \times \ldots \times \mathbf{u})_{n}\right\|^{\frac{p+1}{p}} .
\end{aligned}
$$

Therefore

$$
\begin{aligned}
\|\mathbf{A} \mathbf{w}\|_{X}^{\frac{p+1}{p}} \leq & \sum_{n}\left|D_{n}(1,1)\left(\mathbf{u}^{p} \times \boldsymbol{\eta}\right)_{n}+\frac{D_{n}(1,2)}{p+1}\left(\mathbf{u}_{\times} \ldots \times \mathbf{u}\right)_{n}\right|^{\frac{p+1}{p}} \\
& +\sum_{n}\left|D_{n}(2,1)\left(\mathbf{u}^{p} \times \boldsymbol{\eta}\right)_{n}+\frac{D_{n}(2,2)}{p+1}(\mathbf{u} \times \ldots \times \mathbf{u})_{n}\right|^{\frac{p+1}{p}} \\
\leq & \sum_{n}\left\|D_{n}^{-1}\right\|_{\infty}^{\frac{p+1}{p}}\left(\|\mathbf{u}\|\left\|^{p}\right\| \boldsymbol{\eta}\left\|+\frac{1}{p+1}\right\| \mathbf{u} \|^{p+1}\right)^{\frac{p+1}{p}} \\
& +\sum_{n}\left\|D_{n}^{-1}\right\|_{\infty}^{\frac{p+1}{p}}\left(\|\mathbf{u}\|^{p}\|\boldsymbol{\eta}\|+\frac{1}{p+1}\|\mathbf{u}\|^{p+1}\right)^{\frac{p+1}{p}} \\
\leq & 2\left(\|\mathbf{u}\|^{p}\|\boldsymbol{\eta}\|+\frac{1}{p+1}\|\mathbf{u}\|^{p+1}\right)^{\frac{p+1}{p}} \sum_{n}\left\|D_{n}^{-1}\right\|_{\infty}^{\frac{p+1}{p}} .
\end{aligned}
$$

This yields that $\mathbf{A} \mathbf{w} \in K$ and thus $\mathbf{A} K \subset K$. Let us see now that the operator $\mathbf{A}$ : $K \rightarrow K$ is continuous. Let $\mathbf{w}=(\boldsymbol{\eta}, \mathbf{u}), \overline{\mathbf{w}}=(\overline{\boldsymbol{\eta}}, \overline{\mathbf{u}}) \in K$. Observe that

$$
\left|\left(\mathbf{u}^{p} \times \boldsymbol{\eta}\right)_{n}-\left(\overline{\mathbf{u}}^{p} \times \overline{\boldsymbol{\eta}}\right)_{n}\right|=\left|\sum_{k_{1}+\ldots+k_{p+1}=n}\left(u_{k_{1}} u_{k_{2}} \ldots u_{k_{p}} \eta_{k_{p+1}}-\bar{u}_{k_{1}} \bar{u}_{k_{2}} \ldots \bar{u}_{k p} \bar{\eta}_{k_{p}+1}\right)\right|
$$




$$
\begin{aligned}
= & \mid \sum_{j=1}^{p} \sum_{k_{1}+\ldots+k_{p+1=n}}\left(u_{k_{j}}-\bar{u}_{k_{j}}\right)\left(\bar{u}_{k_{1}} \bar{u}_{k_{2}} \ldots \bar{u}_{k_{j-1}} u_{k_{j+1}} \ldots u_{k_{p}} \eta_{k_{p+1}}\right) \\
& +\left(\eta_{k_{p+1}}-\bar{\eta}_{k p+1}\right) \cdot\left(\bar{u}_{k_{1}} \bar{u}_{k_{2}} \ldots . \bar{u}_{k_{p}}\right)\left|\leq\|\mathbf{u}-\overline{\mathbf{u}}\|\left(\sum_{j=1}^{p}\|\mathbf{u}\|^{p-j}\|\overline{\mathbf{u}}\|^{j-1}\|\boldsymbol{\eta}\|\right)+\|\boldsymbol{\eta}-\overline{\boldsymbol{\eta}}\|\|\mid \overline{\mathbf{u}}\|^{p} .\right.
\end{aligned}
$$

On the other hand,

$$
\begin{aligned}
& \left|(\mathbf{u} \times \ldots . . \times \mathbf{u})_{n}-(\overline{\mathbf{u}} \times \ldots . . \times \overline{\mathbf{u}})_{n}\right| \\
= & \mid \sum_{k_{1}+\ldots . k_{p+1=n}}\left(u_{k_{1}} u_{k_{2}} \ldots . u_{k_{p+1}}-\bar{u}_{k_{1}} \bar{u}_{k_{2}} \ldots . \bar{u}_{k_{p+1}} \mid\right. \\
= & \left|\sum_{j=1}^{p+1} \sum_{k_{1}+\ldots . k_{p+1=n}}\left(u_{k_{j}}-\bar{u}_{k_{j}}\right)\left(\bar{u}_{k_{1}} \bar{u}_{k_{2}} \ldots \bar{u}_{k_{j-1}} u_{k_{j+1}} \ldots u_{k_{p+1}}\right)\right| \\
\leq & \|\mathbf{u}-\overline{\mathbf{u}}\| \sum_{j=1}^{p+1}\|\mathbf{u}\|^{p+1-j}\|\overline{\mathbf{u}}\|^{j-1} .
\end{aligned}
$$

With these results in mind, we have that

$$
\begin{aligned}
& \|\mathbf{A} \mathbf{w}-\mathbf{A} \overline{\mathbf{w}}\|_{X}^{\frac{p+1}{p}} \\
\leq & \sum_{n}\left|D_{n}(1,1)\left(\left(\mathbf{u}^{p} \times \boldsymbol{\eta}\right)_{n}-\left(\overline{\mathbf{u}}^{p} \times \overline{\boldsymbol{\eta}}\right)_{n}\right)+\frac{D_{n}(1,2)}{p+1}\left((\mathbf{u} \times \ldots \times \mathbf{u})_{n}-(\overline{\mathbf{u}} \times \ldots \times \overline{\mathbf{u}})_{n}\right)\right|^{\frac{p+1}{p}} \\
& +\sum_{n}\left|D_{n}(2,1)\left(\left(\mathbf{u}^{p} \times \boldsymbol{\eta}\right)_{n}-\left(\overline{\mathbf{u}}^{p} \times \overline{\boldsymbol{\eta}}\right)_{n}\right)+\frac{D_{n}(2,2)}{p+1}\left((\mathbf{u} \times \ldots \times \mathbf{u})_{n}-(\overline{\mathbf{u}} \times \ldots \times \overline{\mathbf{u}})_{n}\right)\right|^{\frac{p+1}{p}} \\
\leq & 2\left(\sum_{n}\left\|D_{n}^{-1}\right\|_{\infty^{\frac{p+1}{p}}}^{p}\right)\left(\|\mathbf{u}-\overline{\mathbf{u}}\| \sum_{j=1}^{p+1}\|\mathbf{u}\|^{p+1-j}\|\overline{\mathbf{u}}\|^{j-1}\right. \\
& \left.+\frac{1}{p+1}\|\mathbf{u}-\overline{\mathbf{u}}\| \sum_{j=1}^{p}\|\mathbf{u}\|^{p-j}\|\overline{\mathbf{u}}\|^{j-1}\|\boldsymbol{\eta}\|+\frac{1}{p+1}\|\boldsymbol{\eta}-\overline{\boldsymbol{\eta}}\|\|\overline{\mathbf{u}}\|^{p}\right)^{\frac{p+1}{p}} .
\end{aligned}
$$

We conclude that the operator $\mathbf{A}$ is continuous at the point $(\overline{\boldsymbol{\eta}}, \overline{\mathbf{u}})$.

To see that $\mathbf{A}$ is compact let $M$ be a bounded set, i.e. $M \subset\left\{\mathbf{w}=(\boldsymbol{\eta}, \mathbf{u}) \in X:\|\mathbf{w}\|_{X} \leq\right.$ $B\}$. For each $N$, let us define the operator

$$
\left(\mathbf{A}_{N} \mathbf{w}\right)_{n}:= \begin{cases}(\mathbf{A w})_{n} & -N \leq n \leq N \\ 0 & \text { otherwise }\end{cases}
$$

We find that $\mathbf{A}_{N}$ is a compact operator with finite range $2 N+1$. On the other hand, for $\mathbf{w}=(\boldsymbol{\eta}, \mathbf{u}) \in M$

$$
\left|\left(\mathbf{A}_{N} \mathbf{w}\right)_{n}-(\mathbf{A w})_{n}\right|=\left|(\mathbf{A w})_{n>N}\right| .
$$

Now if $(\mathbf{A w})_{n}=\left(v_{n}, z_{n}\right)$, it follows that

$$
\left|v_{n}\right| \leq\left|D_{n}(1,1)\left(\mathbf{u}^{p} \times \boldsymbol{\eta}\right)_{n}+\frac{D_{n}(1,2)}{p+1}(\mathbf{u} \times \ldots \times \mathbf{u})_{n}\right|,
$$




$$
\left|z_{n}\right| \leq\left|D_{n}(2,1)\left(\mathbf{u}^{p} \times \boldsymbol{\eta}\right)_{n}+\frac{D_{n}(2,2)}{p+1}(\mathbf{u} \times \ldots \times \mathbf{u})_{n}\right|,
$$

and therefore

$$
\begin{aligned}
& \left|v_{n}\right| \leq\left\|D_{n}^{-1}\right\|_{\infty}\left(\|\mathbf{u}\|^{p}\|\boldsymbol{\eta}\|+\frac{1}{p+1}\|\mathbf{u}\|^{p+1}\right) \\
& \left|z_{n}\right| \leq\left\|D_{n}^{-1}\right\|_{\infty}\left(\|\mathbf{u}\|^{p}\|\boldsymbol{\eta}\|+\frac{1}{p+1}\|\mathbf{u}\|^{p+1}\right)
\end{aligned}
$$

Then we have the following estimate:

$$
\begin{aligned}
\left\|\mathbf{A}_{N} \mathbf{w}-\mathbf{A} \mathbf{w}\right\|_{X}^{\frac{p+1}{p}} & =\left\|(\mathbf{A} \mathbf{w})_{n>N}\right\|_{X}^{\frac{p+1}{p}} \\
& =\left\|\left(v_{n}\right)_{n>N}\right\|^{\frac{p+1}{p}}+\left\|\left(z_{n}\right)_{n>N}\right\|^{\frac{p+1}{p}} \\
& =\sum_{n>N}\left|v_{n}\right|^{\frac{p+1}{p}}+\sum_{n>N}\left|z_{n}\right|^{\frac{p+1}{p}} \\
& \leq 2 \sum_{n \geq N}\left\|D_{n}^{-1}\right\|_{\infty}^{\frac{p+1}{p}}\left(\|\mathbf{u}\|^{p}\|\boldsymbol{\eta}\|+\frac{1}{p+1}\|\mathbf{u}\|^{p+1}\right)^{\frac{p+1}{p}} \\
& \leq 2\left(\sum_{n \geq N}\left\|D_{n}^{-1}\right\|_{\infty}^{\frac{p+1}{p}}\right)\left(\|\mathbf{u}\|^{p}\|\boldsymbol{\eta}\|+\frac{1}{p+1}\|\mathbf{u}\|^{p+1}\right)^{\frac{p+1}{p}} \\
& \leq 2\left(\sum_{n \geq N}\left\|D_{n}^{-1}\right\|_{\infty}^{\frac{p+1}{p}}\right)\left(B^{p} B+\frac{1}{p+1} B^{p+1}\right)^{\frac{p+1}{p}} \\
& \leq 2\left(\sum_{n \geq N}\left\|D_{n}^{-1}\right\|_{\infty}^{\frac{p+1}{p}}\right) 2^{\frac{p+1}{p}} B^{\frac{(p+1)^{2}}{p}} \\
& \leq 2^{\frac{2 p+1}{p}} \gamma_{N}^{\frac{p+1}{p}} B^{\frac{(p+1)^{2}}{p}} \leq 2^{\frac{2 p+1}{p}} \gamma^{\frac{p+1}{p}} B^{\frac{(p+1)^{2}}{p}}
\end{aligned}
$$

where

$$
\gamma:=\left(\sum_{n}\left\|D_{n}^{-1}\right\|_{\infty}^{\frac{p+1}{p}}\right)^{\frac{p}{p+1}} \text { and } \quad \gamma_{N}:=\left(\sum_{n \geq N}\left\|D_{n}^{-1}\right\|_{\infty}^{\frac{p+1}{p}}\right)^{\frac{p}{p+1}}
$$

Since $\gamma_{N} \rightarrow 0$ as $N \rightarrow \infty$, it follows that $\sup _{\mathbf{w} \in M}\left\|\mathbf{A}_{N} \mathbf{w}-\mathbf{A} \mathbf{w}\right\|_{X}^{\frac{p+1}{p}} \rightarrow 0, N \rightarrow \infty$. Then $\mathbf{A}$ is a compact operator because it is the uniform limit of compact operators on bounded sets. This concludes the proof of the lemma.

LEMma 5.2. Let $\gamma$ be as defined in (5.3). Then for any $r$ satisfying

$$
0<r<r_{0}:=\min \left\{\left\|\left(\boldsymbol{p}^{*}, \boldsymbol{q}^{*}\right)\right\|_{X}, \frac{1}{\gamma^{\frac{1}{p}} 2^{\frac{2 p+1}{p(p+1)}}}\right\},
$$

$\boldsymbol{w} \neq t \boldsymbol{A} \boldsymbol{w}$ for all $\boldsymbol{w} \in \partial K_{r}$ and for all $t \in[0,1]$.

Proof. Suppose that there exists $\mathbf{w}=(\boldsymbol{\eta}, \mathbf{u})=\left(\eta_{n}, u_{n}\right) \in \partial K_{r}$ and $t \in[0,1]$ such that $\mathbf{w}=t \mathbf{A w}$. Then using (5.1), we see that

$$
\|\mathbf{w}\|_{X}^{\frac{p+1}{p}}=\|t \mathbf{A} \mathbf{w}\|_{X}^{\frac{p+1}{p}} \leq\|\mathbf{A} \mathbf{w}\|_{X}^{\frac{p+1}{p}}=\left\|\left(\eta_{n}\right)\right\|^{\frac{p+1}{p}}+\left\|\left(u_{n}\right)\right\|^{\frac{p+1}{p}}
$$


where $(\mathbf{A w})_{n}=\left(v_{n}, z_{n}\right)$. Therefore

$$
\begin{aligned}
& r^{\frac{p+1}{p}}=\|\mathbf{w}\|_{X}^{\frac{p+1}{p}} \leq \sum_{n}\left|v_{n}\right|^{\frac{p+1}{p}}+\sum_{n}\left|z_{n}\right|^{\frac{p+1}{p}} \\
& \leq 2\left(\sum_{n}\left\|D_{n}^{-1}\right\|_{\infty}^{\frac{p+1}{p}}\right)\left(\|\mathbf{u}\|^{p}\|\boldsymbol{\eta}\|+\frac{1}{p+1}\|\mathbf{u}\|^{p+1}\right)^{\frac{p+1}{p}} \\
& \leq 2 \gamma^{\frac{p+1}{p}}\left(r^{p+1}+\frac{1}{p+1} r^{p+1}\right)^{\frac{p+1}{p}} \\
& \leq 2^{\frac{2 p+1}{p}} r^{\frac{(p+1)^{2}}{p}} \gamma^{\frac{p+1}{p}},
\end{aligned}
$$

from which follows that

$$
2^{-\frac{2 p+1}{p(p+1)}} \gamma^{-\frac{1}{p}} \leq r
$$

contradicting the choice of $r$.

Lemma 5.3. For any

$$
R>R_{0}:=\max \left\{\left\|\left(\boldsymbol{p}^{*}, \boldsymbol{q}^{*}\right)\right\|_{X}, \frac{2^{\frac{p}{p+1}}(p+1)^{\frac{1}{p}}\left(c^{2}-1\right)^{1 / p}\left(\left(c^{2}-1\right) \gamma+\beta\right)}{c^{1 / p}}\right\},
$$

with

$$
\beta=\left(\sum_{n} \frac{1}{\left(1+n^{2}\right)^{\frac{p+1}{p}}}\right)^{\frac{p}{p+1}}
$$

there exists a non-zero $\overline{\mathbf{w}} \in K$ such that $\mathbf{w}-\mathbf{A} \mathbf{w} \neq \lambda \overline{\mathbf{w}}$, for all $\mathbf{w} \in \partial K_{R}$ and all $\lambda \geq 0$.

Proof. Let $\overline{\mathbf{w}}=(\overline{\boldsymbol{\eta}}, \overline{\mathbf{u}})=\left(\bar{\eta}_{n}, \bar{u}_{n}\right)$ be given by

$$
\left(\begin{array}{l}
\bar{\eta}_{n} \\
\bar{u}_{n}
\end{array}\right)=\frac{1}{1+n^{2}}\left(\begin{array}{l}
1 \\
1
\end{array}\right) \neq 0 \text {. }
$$

Then it is clear that $\overline{\mathbf{w}} \in K$. Suppose that there exists $\mathbf{w}=(\boldsymbol{\eta}, \mathbf{u}) \in \partial K_{R}$ and $\lambda \geq 0$ such that for all $n$,

$$
\left(\begin{array}{l}
\eta_{n} \\
u_{n}
\end{array}\right)=D_{n}^{-1}\left(\begin{array}{c}
\left(\mathbf{u}^{p} \times \boldsymbol{\eta}\right)_{n} \\
\frac{1}{p+1}(\mathbf{u} \times \ldots \times \mathbf{u})_{n}
\end{array}\right)+\lambda\left(\begin{array}{l}
\bar{\eta}_{n} \\
\bar{u}_{n}
\end{array}\right) .
$$

In particular,

$$
\begin{aligned}
\eta_{0} & =\frac{1}{c^{2}-1}\left(c\left(\mathbf{u}^{p} \times \boldsymbol{\eta}\right)_{0}+\frac{1}{p+1}(\mathbf{u} \times \ldots \times \mathbf{u})_{0}\right)+\lambda, \\
u_{0} & =\frac{1}{c^{2}-1}\left(\left(\mathbf{u}^{p} \times \boldsymbol{\eta}\right)_{0}+\frac{c}{p+1}(\mathbf{u} \times \ldots \times \mathbf{u})_{0}\right)+\lambda .
\end{aligned}
$$

Firstly note that if $\eta_{0}=0$ then $\eta_{n}=0$ for all $n$, and from Equation (5.5) it follows that

$$
\frac{u_{0}^{p+1}}{\left(c^{2}-1\right)(p+1)}+\lambda=0
$$


and therefore $u_{0}=0$ since $\lambda, u_{0} \geq 0$. Similarly, $u_{0}=0$ implies that $\eta_{0}=0$. Therefore since $\mathbf{w}=\mathbf{0} \notin \partial K_{R}$ we find that $\eta_{0} \neq 0$ and $u_{0} \neq 0$. Consequently from Equation (5.5), it follows that

$$
u_{0} \geq \frac{1}{c^{2}-1}\left(\frac{c u_{0}^{p+1}}{p+1}\right)
$$

and therefore we found the bound

$$
0<u_{0} \leq\left(\frac{\left(c^{2}-1\right)(p+1)}{c}\right)^{\frac{1}{p}}
$$

On the other hand, since

$$
u_{0} \geq \frac{1}{c^{2}-1}\left(\mathbf{u}^{p} \times \boldsymbol{\eta}\right)_{0} \geq \frac{1}{c^{2}-1} u_{0}^{p} \eta_{0},
$$

we have that $0<\eta_{0} \leq\left(c^{2}-1\right)^{1 / p} c^{(p-1) / p}(p+1)^{(1-p) / p}$. Furthermore

$$
0 \leq \lambda \leq u_{0} \leq\left(c^{2}-1\right)^{1 / p}(p+1)^{1 / p} c^{-1 / p} .
$$

One can see from Equation (5.4) and from the fact that $t_{n}, s_{n}$ are decreasing in $|n|$ that

$$
\begin{aligned}
& \eta_{n} \leq\left\|D_{n}^{-1}\right\|_{\infty}\left(\left(\mathbf{u}^{p} \times \boldsymbol{\eta}\right)_{0}+\frac{1}{p+1}(\mathbf{u} \times \ldots \times \mathbf{u})_{0}\right)+\frac{\lambda}{1+n^{2}}, \\
& u_{n} \leq\left\|D_{n}^{-1}\right\|_{\infty}\left(\left(\mathbf{u}^{p} \times \boldsymbol{\eta}\right)_{0}+\frac{1}{p+1}(\mathbf{u} \times \ldots \times \mathbf{u})_{0}\right)+\frac{\lambda}{1+n^{2}} .
\end{aligned}
$$

We remark also that

$$
\frac{1}{c^{2}-1}\left(\left(\mathbf{u}^{p} \times \boldsymbol{\eta}\right)_{0}+\frac{1}{p+1}(\mathbf{u} \times \ldots \times \mathbf{u})_{0}\right) \leq u_{0} \leq\left(c^{2}-1\right)^{1 / p}(p+1)^{1 / p} c^{-1 / p},
$$

since $c>1$. Therefore

$$
\left(\mathbf{u}^{p} \times \boldsymbol{\eta}\right)_{0}+\frac{1}{p+1}(\mathbf{u} \times \ldots \times \mathbf{u})_{0} \leq\left(c^{2}-1\right)^{\frac{p+1}{p}}(p+1)^{\frac{1}{p}} c^{-\frac{1}{p}} .
$$

As a consequence,

$$
\begin{aligned}
& \eta_{n} \leq\left\|D_{n}^{-1}\right\|_{\infty}\left(c^{2}-1\right)^{\frac{p+1}{p}}(p+1)^{\frac{1}{p}} c^{-\frac{1}{p}}+\left(c^{2}-1\right)^{\frac{1}{p}}(p+1)^{\frac{1}{p}} c^{-\frac{1}{p}} \frac{1}{1+n^{2}}, \\
& u_{n} \leq\left\|D_{n}^{-1}\right\|_{\infty}\left(c^{2}-1\right)^{\frac{p+1}{p}}(p+1)^{\frac{1}{p}} c^{-\frac{1}{p}}+\left(c^{2}-1\right)^{\frac{1}{p}}(p+1)^{\frac{1}{p}} c^{-\frac{1}{p}} \frac{1}{1+n^{2}} .
\end{aligned}
$$

From the fact that $\mathbf{w}=(\boldsymbol{\eta}, \mathbf{u}) \in \partial K_{R}$, we deduce that

$$
\begin{aligned}
R^{\frac{p+1}{p}} & =\sum_{n}\left|\eta_{n}\right|^{\frac{p+1}{p}}+\sum_{n}\left|u_{n}\right|^{\frac{p+1}{p}} \\
& \leq 2(p+1)^{\frac{p+1}{p^{2}}} c^{-\frac{p+1}{p^{2}}}\left(c^{2}-1\right)^{\frac{p+1}{p^{2}}} \sum_{n}\left(\left\|D_{n}^{-1}\right\|_{\infty}\left(c^{2}-1\right)+\frac{1}{1+n^{2}}\right)^{\frac{p+1}{p}} .
\end{aligned}
$$

The triangle inequality for summations implies that

$$
R^{\frac{p+1}{p}} \leq 2(p+1)^{\frac{p+1}{p^{2}}} c^{-\frac{p+1}{p^{2}}}\left(c^{2}-1\right)^{\frac{p+1}{p^{2}}}\left[\left(\sum_{n}\left\|D_{n}^{-1}\right\|_{\infty}^{\frac{p+1}{p}}\left(c^{2}-1\right)^{\frac{p+1}{p}}\right)^{\frac{p}{p+1}}+H\right]^{\frac{p+1}{p}},
$$


where

$$
H:=\left(\sum_{n}\left(\frac{1}{1+n^{2}}\right)^{\frac{p+1}{p}}\right)^{\frac{p}{p+1}}
$$

Therefore,

$$
R \leq 2^{\frac{p}{p+1}}(p+1)^{\frac{1}{p}} c^{-\frac{1}{p}}\left(c^{2}-1\right)^{\frac{1}{p}}\left(\left(c^{2}-1\right) \gamma+\beta\right),
$$

which contradicts the choice of the radius $R$.

Theorem 5.4. Let $r$ and $R$ be as above. Then the fixed point index of $\boldsymbol{A}$ on $K_{r}^{R}=$ $\left\{\boldsymbol{w} \in K: r<\|\boldsymbol{w}\|_{X}<R\right\}$ is $i\left(K, \boldsymbol{A}, K_{r}^{R}\right)=-1$.

Proof. This is a consequence of Lemmas 5.2 and 5.3 and Theorem 3.4.

REMARK 5.5. In order to complete the analysis presented above, we have to establish the existence of a non-trivial fixed point of the operator $\mathbf{A}$ different from the constant periodic solution $\left(\mathbf{p}^{*}, \mathbf{q}^{*}\right)$ which belongs to $K_{r}^{R}$. To address this issue let us define the sets

$$
\begin{gathered}
K_{\epsilon}\left(\mathbf{p}^{*}, \mathbf{q}^{*}\right):=\left\{\mathbf{w}=(\boldsymbol{\eta}, \mathbf{u}) \in K:\left\|(\boldsymbol{\eta}, \mathbf{u})-\left(\mathbf{p}^{*}, \mathbf{q}^{*}\right)\right\|_{X}<\epsilon\right\}, \\
\partial K_{\epsilon}\left(\mathbf{p}^{*}, \mathbf{q}^{*}\right):=\left\{\mathbf{w}=(\boldsymbol{\eta}, \mathbf{u}) \in K:\left\|(\boldsymbol{\eta}, \mathbf{u})-\left(\mathbf{p}^{*}, \mathbf{q}^{*}\right)\right\|_{X}=\epsilon\right\},
\end{gathered}
$$

for $\epsilon>0$.

Lemma 5.6. If $\left(\boldsymbol{p}^{*}, \boldsymbol{q}^{*}\right)$ is the only fixed point of $\boldsymbol{A}$ in $K_{r}^{R}$, then when the half-period $l>0$ is chosen large enough then we have $i\left(K, \mathbf{A}, K_{\epsilon}\left(\boldsymbol{p}^{*}, \boldsymbol{q}^{*}\right)\right)=0$.

Proof. The lemma is proved due to Lemma 3.3, if we can show that ( $I-$ A) $\partial K_{\epsilon}\left(\mathbf{p}^{*}, \mathbf{q}^{*}\right)$ is disjoint with the set $\{\lambda \overline{\mathbf{w}}: \lambda \geq 0\}$, where $\overline{\mathbf{w}} \in K$ was defined in Lemma 5.3. Suppose that there are $\mathbf{w}=(\boldsymbol{\eta}, \mathbf{u}) \in \partial K_{\epsilon}\left(\mathbf{p}^{*}, \mathbf{q}^{*}\right)$ and $\lambda \geq 0$ such that $\mathbf{w}-\mathbf{A w}=$ $\lambda \overline{\mathbf{w}}$. Then for all $n \in \mathbb{Z}$ we have that

$$
\left(\begin{array}{l}
\eta_{n} \\
u_{n}
\end{array}\right)=\frac{1}{\operatorname{det} D_{n}^{-1}}\left(\begin{array}{cc}
c\left(1-\frac{\delta n^{2} \pi^{2}}{l^{2}}\right) & 1-(\theta-\mu c) \frac{n^{2} \pi^{2}}{l^{2}} \\
1-(\theta-\mu c) \frac{n^{2} \pi^{2}}{l^{2}} & c\left(1-\frac{\delta n^{2} \pi^{2}}{l^{2}}\right)
\end{array}\right)\left(\begin{array}{c}
\left(\mathbf{u}^{p} \times \boldsymbol{\eta}\right)_{n} \\
\frac{1}{p+1}(\mathbf{u} \times \ldots \times \mathbf{u})_{n}
\end{array}\right)+\lambda \overline{\mathbf{w}} .
$$

For $n=1$ the equation above implies that

$$
\begin{aligned}
& \eta_{1}=\frac{1}{\operatorname{det} D_{1}^{-1}}\left(c\left(1-\frac{\delta \pi^{2}}{l^{2}}\right)\left(\mathbf{u}^{p} \times \boldsymbol{\eta}\right)_{1}+(1-(\theta-\mu c)) \frac{\pi^{2}}{l^{2}} \frac{1}{p+1}(\mathbf{u} \times \ldots \times \mathbf{u})_{1}\right)+\frac{\lambda}{2}, \\
& u_{1}=\frac{1}{\operatorname{det} D_{1}^{-1}}\left(\left(1-(\theta-\mu c) \frac{\pi^{2}}{l^{2}}\right)\left(\mathbf{u}^{p} \times \boldsymbol{\eta}\right)_{1}+\frac{c}{p+1}\left(1-\frac{\delta \pi^{2}}{l^{2}}\right)(\mathbf{u} \times \ldots \times \mathbf{u})_{1}\right)+\frac{\lambda}{2} .
\end{aligned}
$$

Therefore

$$
\begin{aligned}
& \eta_{1} \geq \frac{1}{\operatorname{det} D_{1}^{-1}} c\left(1-\frac{\delta \pi^{2}}{l^{2}}\right)\left(u_{0}^{p} \eta_{1}+u_{1} \eta_{0}^{p}\right)+\frac{1}{\operatorname{det} D_{1}^{-1}}\left(1-(\theta-\mu c) \frac{\pi^{2}}{l^{2}}\right) u_{0}^{p} u_{1}+\frac{\lambda}{2}, \\
& u_{1} \geq \frac{1}{\operatorname{det} D_{1}^{-1}}\left(1-(\theta-\mu c) \frac{\pi^{2}}{l^{2}}\right)\left(u_{0}^{p} \eta_{1}+u_{1} \eta_{0}^{p}\right)+\frac{1}{\operatorname{det} D_{1}^{-1}} c\left(1-\frac{\delta \pi^{2}}{l^{2}}\right) u_{0}^{p} u_{1}+\frac{\lambda}{2} .
\end{aligned}
$$


As a consequence, we obtain

$$
\begin{aligned}
& \eta_{1} \geq A\left(u_{0}^{p} \eta_{1}+u_{1} \eta_{0}^{p}\right)+B u_{0}^{p} u_{1}+\frac{\lambda}{2}, \\
& u_{1} \geq B\left(u_{0}^{p} \eta_{1}+u_{1} \eta_{0}^{p}\right)+A u_{0}^{p} u_{1}+\frac{\lambda}{2},
\end{aligned}
$$

where

$$
\begin{aligned}
& A=\frac{1}{\operatorname{det} D_{1}^{-1}} c\left(1-\frac{\delta \pi^{2}}{l^{2}}\right) \\
& B=\frac{1}{\operatorname{det} D_{1}^{-1}}\left(1-(\theta-\mu c) \frac{\pi^{2}}{l^{2}}\right) .
\end{aligned}
$$

It follows that

$$
\begin{aligned}
\eta_{1}+u_{1} & \geq B u_{0}^{p}\left(\eta_{1}+u_{1}\right)+A \cdot u_{0}^{p}\left(\eta_{1}+u_{1}\right)+u_{1} \eta_{0}^{p}(A+B)+\lambda \\
& \geq(A+B) u_{0}^{p}\left(\eta_{1}+u_{1}\right)+(A+B) u_{1} \eta_{0}^{p}+\lambda .
\end{aligned}
$$

Since $\mathbf{w} \in \partial K_{\epsilon}\left(\mathbf{p}^{*}, \mathbf{q}^{*}\right)$, we can write

$$
\mathbf{w}=(\boldsymbol{\eta}, \mathbf{u})=\left(\mathbf{p}^{*}, \mathbf{q}^{*}\right)+\epsilon(\tilde{\boldsymbol{\eta}}, \tilde{\mathbf{u}}),
$$

with $\|(\tilde{\boldsymbol{\eta}}, \tilde{\mathbf{u}})\|_{X}=1$. Note that for $n \geq 1$,

$$
\tilde{\eta}_{n}=\eta_{n} / \epsilon \geq 0, \quad \tilde{u}_{n}=u_{n} / \epsilon \geq 0,
$$

and $\tilde{\eta}_{n} \geq \tilde{\eta}_{n+1}, \tilde{u}_{n} \geq \tilde{u}_{n+1}$. Further we have

$$
\eta_{0}=p_{0}+\epsilon \tilde{\eta}_{0}, \quad u_{0}=q_{0}+\epsilon \tilde{u}_{0} .
$$

Then in terms of the new variables $(\tilde{\boldsymbol{\eta}}, \tilde{\mathbf{u}})$, Equation (5.8) can be written as

$$
\epsilon\left(\tilde{\eta}_{1}+\tilde{u}_{1}\right) \geq \epsilon(A+B)\left(q_{0}+\epsilon \tilde{u}_{0}\right)^{p}\left(\tilde{\eta}_{1}+\tilde{u}_{1}\right)+\epsilon(A+B) \tilde{u}_{1}\left(p_{0}+\epsilon \tilde{\eta}_{0}\right)^{p}+\lambda .
$$

Now observe that

$$
q_{0}=\left(\frac{c(p+2)-\sqrt{p^{2} c^{2}+4 p+4}}{2}\right)>(c-1)^{\frac{1}{p}},
$$

and thus the equation $p_{0}=\frac{q_{0}}{c-q_{0}^{p}}$ implies that $p_{0}>q_{0}>(c-1)^{\frac{1}{p}}$. Therefore the halfperiod $l>0$ can be selected large enough in order to ensure that

$$
q_{0}>(A+B)^{-1 / p}=\left(c-1+(\theta-\mu c-\delta c) \frac{\pi^{2}}{l^{2}}\right)^{1 / p}
$$

and

$$
p_{0}>(A+B)^{-1 / p}=\left(c-1+(\theta-\mu c-\delta c) \frac{\pi^{2}}{l^{2}}\right)^{1 / p} .
$$

The number $\epsilon>0$ can be selected small enough to guarantee that

$$
u_{0}=q_{0}+\epsilon \tilde{u}_{0}>(A+B)^{-1 / p}>0, \quad \eta_{0}=p_{0}+\epsilon \tilde{\eta}_{0}>(A+B)^{-1 / p}>0 .
$$


With this choice of $\epsilon$, it follows from inequality (5.9) that

$$
\epsilon\left(\tilde{\eta}_{1}+\tilde{u}_{1}\right) \geq \epsilon\left(\tilde{\eta}_{1}+\tilde{u}_{1}\right)+\epsilon \tilde{u}_{1}+\lambda .
$$

Therefore,

$$
0 \geq \epsilon \tilde{u}_{1}+\lambda
$$

Furthermore we know that $\tilde{u}_{1} \geq 0$ and $\lambda \geq 0$. We conclude that $\tilde{u}_{1}=\lambda=0$ and thus $\tilde{u}_{n}=0$ for all $n \neq 0$. Observe that Equation (5.6) for $n=0$ reads

$$
\begin{aligned}
& \eta_{0}=\frac{c}{c^{2}-1} u_{0}^{p} \eta_{0}+\frac{1}{(p+1)\left(c^{2}-1\right)} u_{0}^{p+1}, \\
& u_{0}=\frac{1}{c^{2}-1} u_{0}^{p} \eta_{0}+\frac{c}{(p+1)\left(c^{2}-1\right)} u_{0}^{p+1} .
\end{aligned}
$$

Therefore, substituting Equation (5.10) into Equation (5.11) yields that

$$
u_{0}=\frac{1}{c^{2}-1} u_{0}^{p} \eta_{0}+c\left(\eta_{0}-\frac{c}{c^{2}-1} u_{0}^{p} \eta_{0}\right),
$$

and thus

$$
u_{0}=\left(c-u_{0}^{p}\right) \eta_{0}
$$

or equivalently,

$$
\eta_{0}=\frac{u_{0}}{c-u_{0}^{p}}
$$

Observe that Equation (5.13) can be rewritten as

$$
\eta_{0}=\frac{c \eta_{0}-u_{0}}{u_{0}^{p}}
$$

As a consequence, introducing the equation above into Equation (5.11) we arrive at

$$
u_{0}=\frac{1}{c^{2}-1} u_{0}^{p}\left(\frac{c \eta_{0}-u_{0}}{u_{0}^{p}}\right)+\frac{c}{\left(c^{2}-1\right)(p+1)} u_{0}^{p+1},
$$

from where we obtain that

$$
\eta_{0}-c u_{0}=-\frac{u_{0}^{p+1}}{p+1}
$$

Equations (5.13) and (5.14) imply that $u_{0}$ and $\eta_{0}$ satisfy the same equations as $q_{0}, p_{0}$, respectively. We conclude that $u_{0}=q_{0}>0$ and $\eta_{0}=p_{0}>0$ and $\tilde{\eta}_{0}=\tilde{u}_{0}=0$.

Now $0=u_{1}=\epsilon \tilde{u}_{1} \geq B u_{0}^{p} \eta_{1}$ (from Equation (5.7)) implies that $\tilde{\eta}_{1}=\eta_{1} / \epsilon=0$. Recall that $A, B$ are positive. This implies that $\tilde{\eta}_{1} \geq \tilde{\eta}_{n}=0$, for all $n \neq 0$. Therefore we have shown that $\tilde{u}_{n}=\tilde{\eta}_{n}=0$ for all $n$, contradicting the fact that $\mathbf{w}=(\boldsymbol{\eta}, \mathbf{u})=\left(\mathbf{p}^{*}, \mathbf{q}^{*}\right)+$ $\epsilon(\tilde{\boldsymbol{\eta}}, \tilde{\mathbf{u}}) \in \partial K_{\epsilon}\left(\mathbf{p}^{*}, \mathbf{q}^{*}\right)$.

THEOREM 5.7. For the wave speed $c$ satisfying the inequality given in (4.8), and if the half-period $l$ is chosen large enough, then the operator $\boldsymbol{A}$ has a non-trivial fixed point $\overline{\boldsymbol{w}}=(\bar{\eta}, \bar{u})$ in the cone segment $K_{r}^{R}$.

Proof. Existence of a non-trivial fixed point of the operator $\mathbf{A}$ follows from Theorem 5.4 and Lemma 5.6. We remark that it can also be shown that the functions

$$
\bar{\eta}(x, t)=\bar{\eta}(x-c t)=\sum_{n} \bar{\eta}_{n} e^{i(n \pi / l)(x-c t)}, \quad \bar{u}(x, t)=\bar{u}(x-c t)=\sum_{n} \bar{u}_{n} e^{i(n \pi / l)(x-c t)}
$$

are infinitely smooth and thus they correspond to a non-trivial periodic travellingwave solution of the original system (4.1). 


\section{Approximation of periodic travelling-wave solutions}

In previous sections we have established existence of periodic travelling-wave solutions of the Boussinesq system (4.1) under some conditions on the wave speed and the model's parameters. Since these solutions can not be expressed analytically when $p>1$, we want to formulate an efficient technique which allows us to approximate them. This will be accomplished by using a pseudospectral numerical solver based on a Fourier decomposition which is an adequate technique for a spatially-periodic framework.
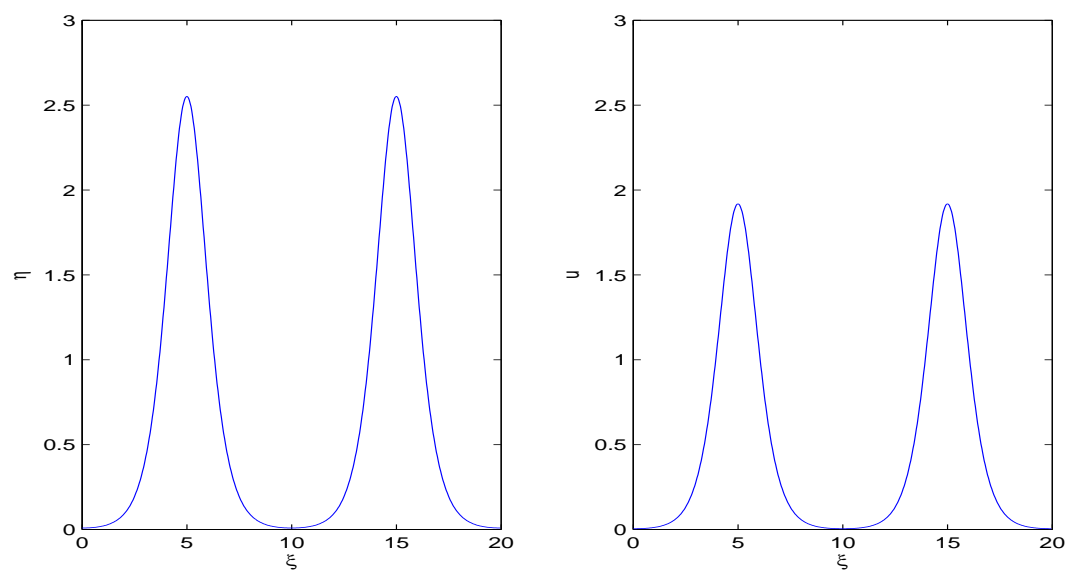

FIG. 6.1. Travelling-wave solution $(\eta, u)$ with fundamental period 10 , wave speed $c=2$, and $p=1$.
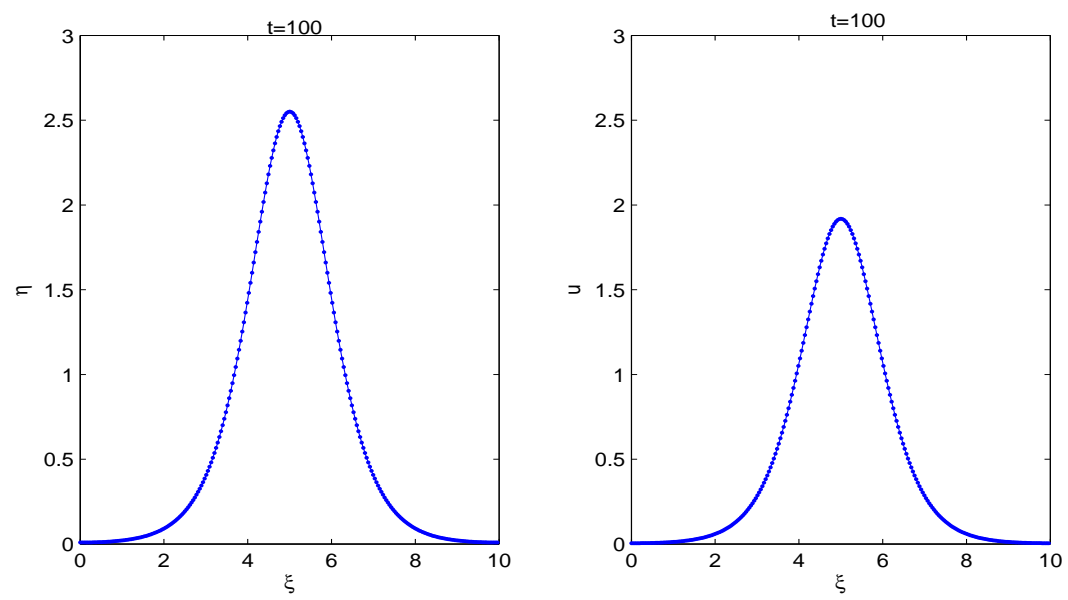

FIG. 6.2. Solid line: Numerical solution of system (4.1) using as initial data the profiles in Figure 6.1. Pointed line: Profiles in Figure 6.1 shifted a distance equal to $100 \mathrm{c}=200$ units to the right. 

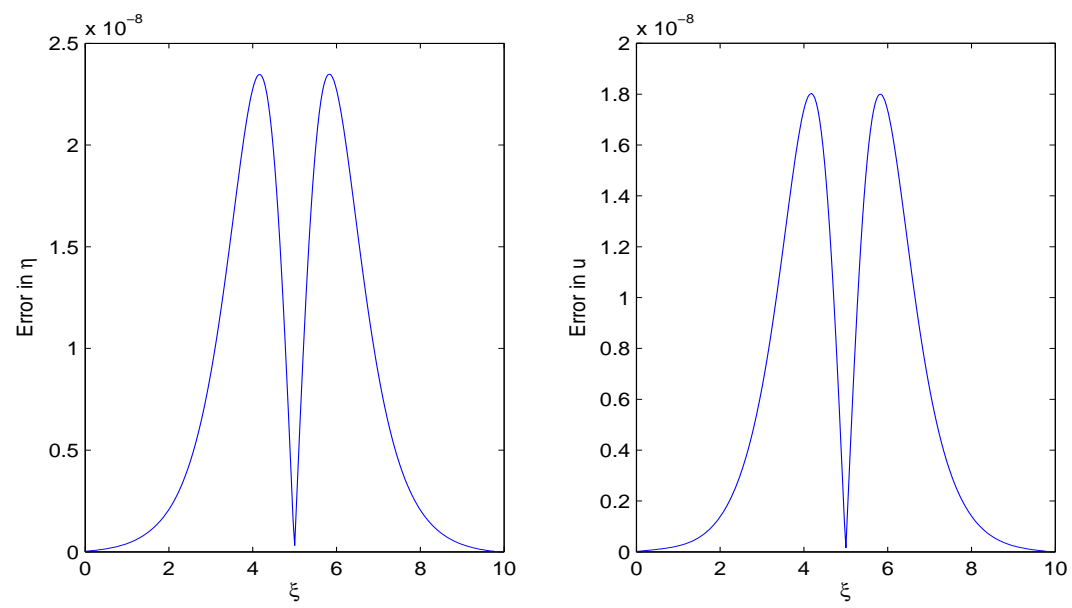

FIG. 6.3. In this figure we plot the absolute value of the difference between the profiles presented in Figure 6.2.
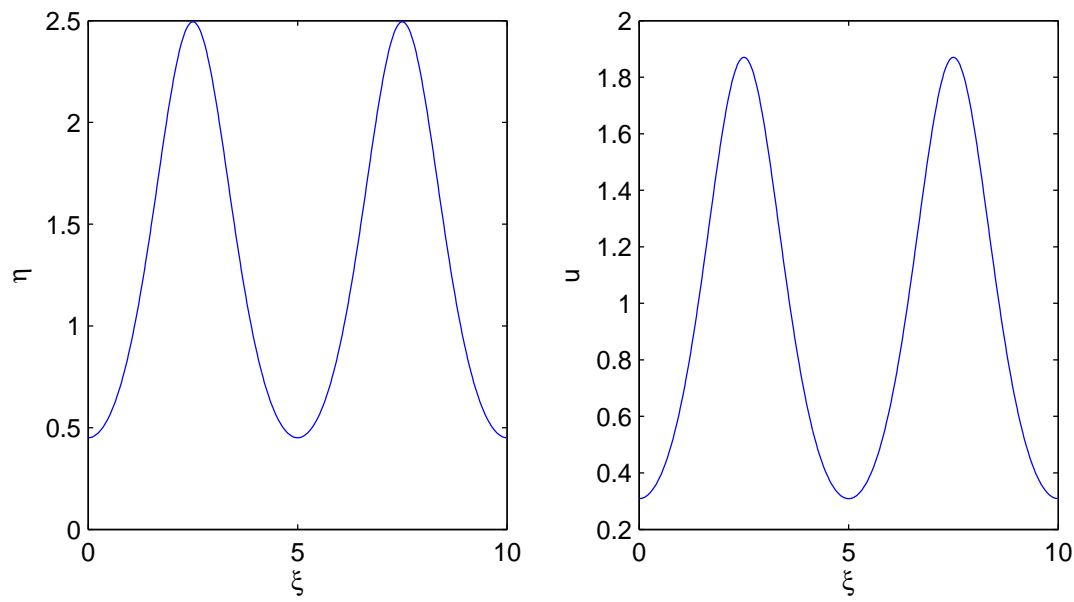

FIG. 6.4. Travelling-wave solution $(\eta, u)$ with fundamental period 5 , wave speed $c=2$, and $p=1$.

Recall that travelling-wave solutions of System (4.1) must satisfy Equation (4.3), which in turn can be rewritten as

$$
\begin{aligned}
& \eta^{\prime \prime}(\xi)=\frac{1}{\Delta}\left(c \delta\left(u-c \eta+u^{p} \eta\right)-(\mu c-\theta)\left(-c u+\eta+\frac{u^{p+1}}{p+1}\right)\right), \\
& u^{\prime \prime}(\xi)=\frac{1}{\Delta}\left(c \delta\left(-c u+\eta+\frac{u^{p+1}}{p+1}\right)-(\mu c-\theta)\left(u-c \eta+u^{p} \eta\right)\right),
\end{aligned}
$$

where $\Delta=(c \delta)^{2}-(\mu c-\theta)^{2}$.

Since we are interested in finding approximations to even solutions $(\eta, u)$ with period $2 l, l>0$ of Equation (6.1), let us introduce truncated cosine expansions for $\eta$ 

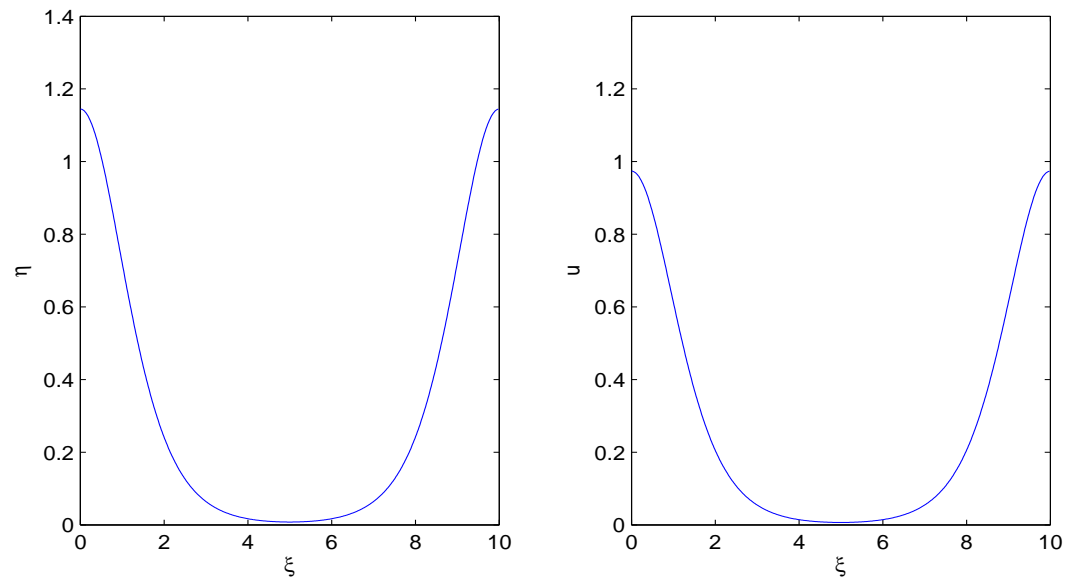

FIG. 6.5. Travelling-wave solution $(\eta, u)$ with half-period $l=5$, wave speed $c=1.5$, and $p=1$.
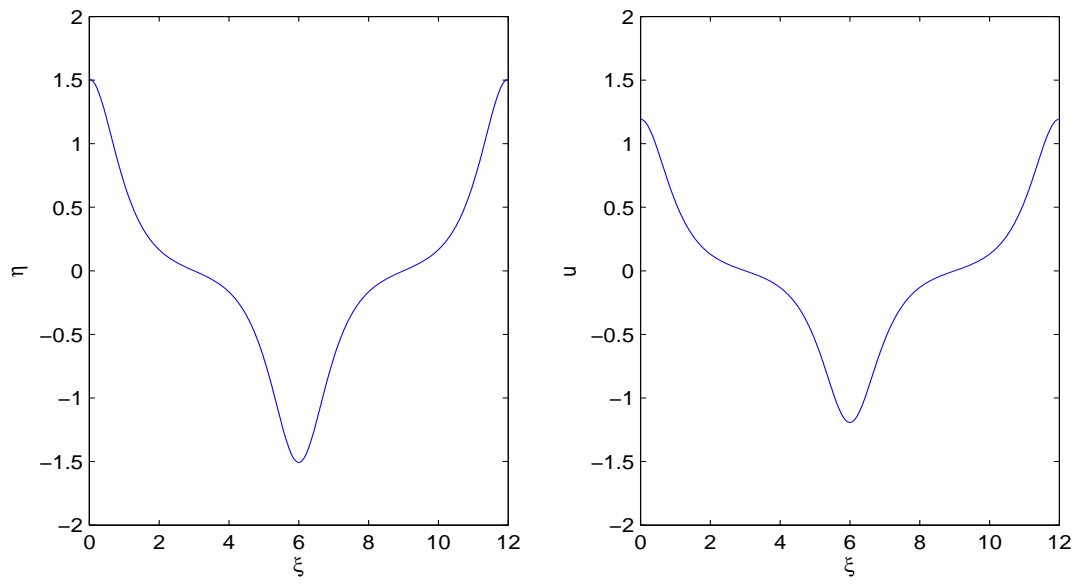

FIG. 6.6. Travelling-wave solution $(\eta, u)$ with half-period $l=6$, wave speed $c=1.5$, and $p=2$.

and $u$ :

$$
\begin{aligned}
& \eta(\xi) \approx \eta_{0}+\sum_{n=1}^{N / 2} \eta_{n} \cos \left(\frac{n \pi}{l} \xi\right), \\
& u(\xi) \approx u_{0}+\sum_{n=1}^{N / 2} u_{n} \cos \left(\frac{n \pi}{l} \xi\right),
\end{aligned}
$$



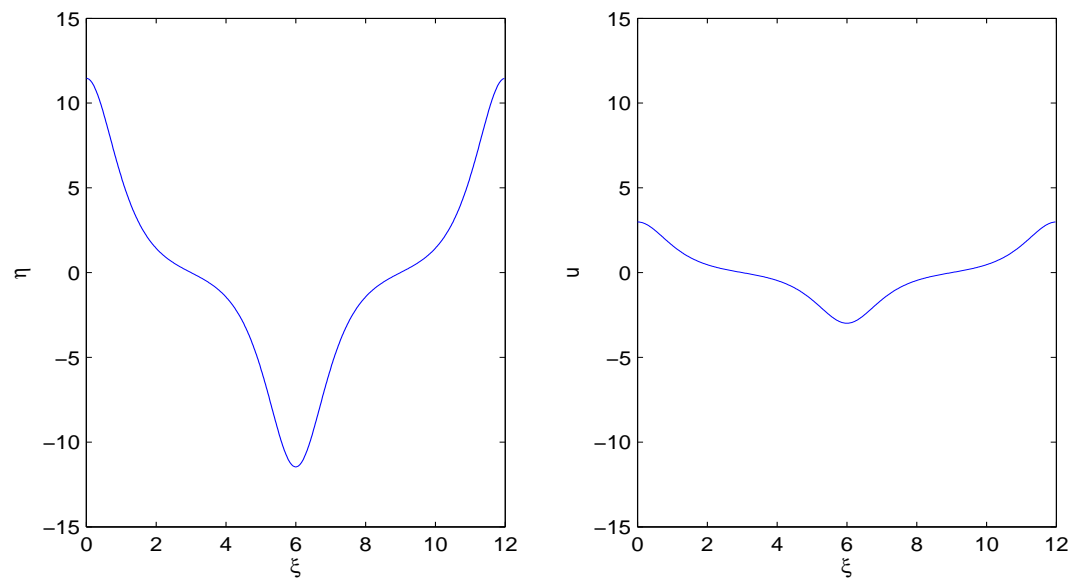

FIG. 6.7. Travelling-wave solution $(\eta, u)$ with half-period $l=6$, wave speed $c=5$, and $p=2$.
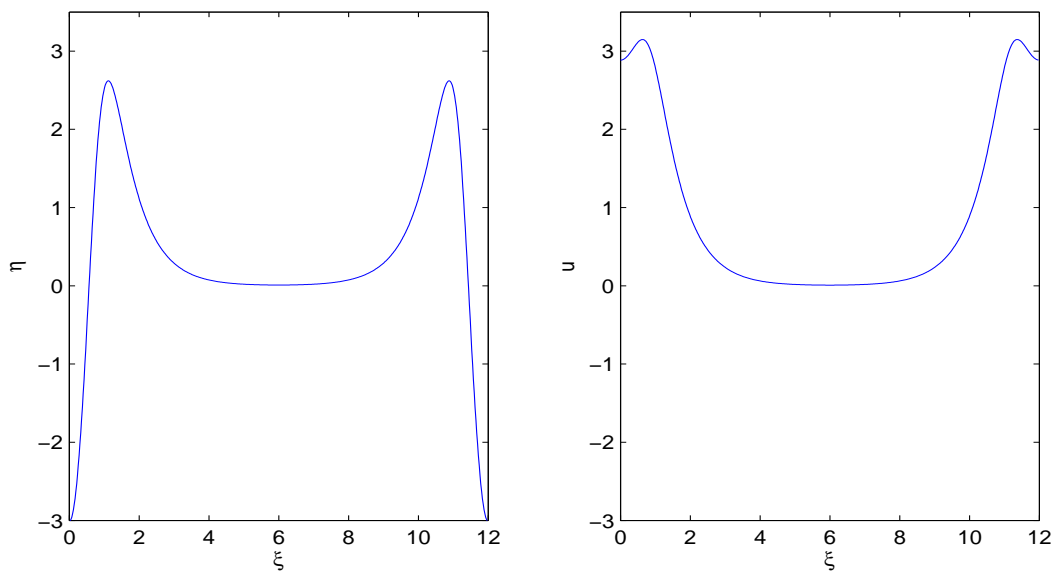

FIG. 6.8. Travelling-wave solution $(\eta, u)$ with half-period $l=6$, wave speed $c=5$, and $p=3$.

where

$$
\begin{aligned}
& \eta_{0}=\frac{1}{l} \int_{0}^{l} \eta(\xi) d \xi=\frac{1}{2 l} \int_{0}^{2 l} \eta(\xi) d \xi \\
& \eta_{n}=\frac{2}{l} \int_{0}^{l} \eta(\xi) \cos \left(\frac{n \pi \xi}{l}\right) d \xi=\frac{2}{2 l} \int_{0}^{2 l} \eta(\xi) \cos \left(\frac{n \pi \xi}{l}\right) d \xi
\end{aligned}
$$

By substituting expansions (6.2) into Equation (6.1) and evaluating them at the $N / 2+1$ collocation points

$$
\xi_{j}=\frac{2 l(j-1)}{N}, \quad j=1, \ldots, N / 2+1,
$$



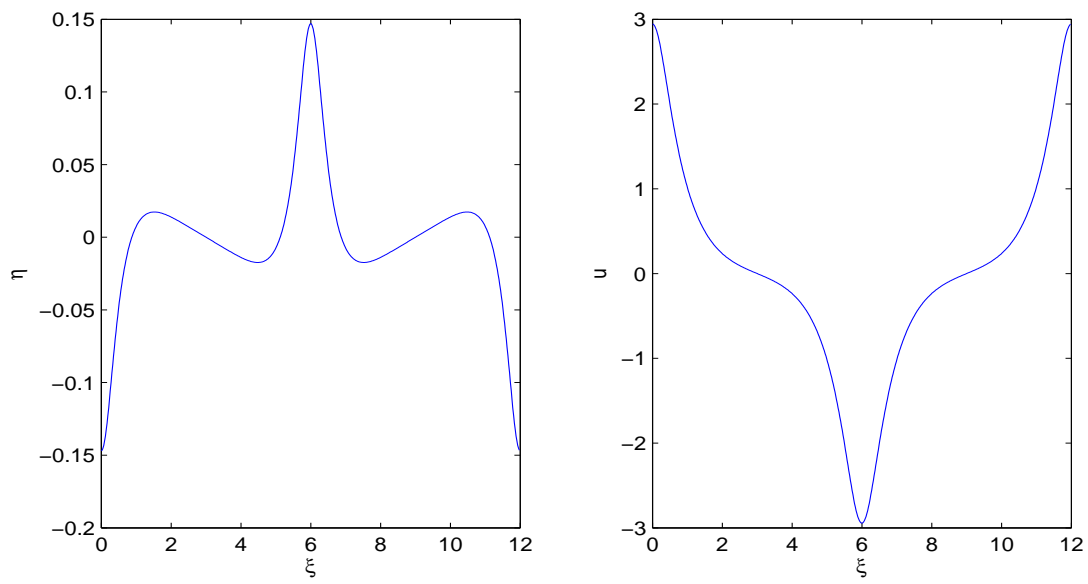

FIG. 6.9. Travelling-wave solution $(\eta, u)$ with half-period $l=6$, wave speed $c=5$, and $p=4$.
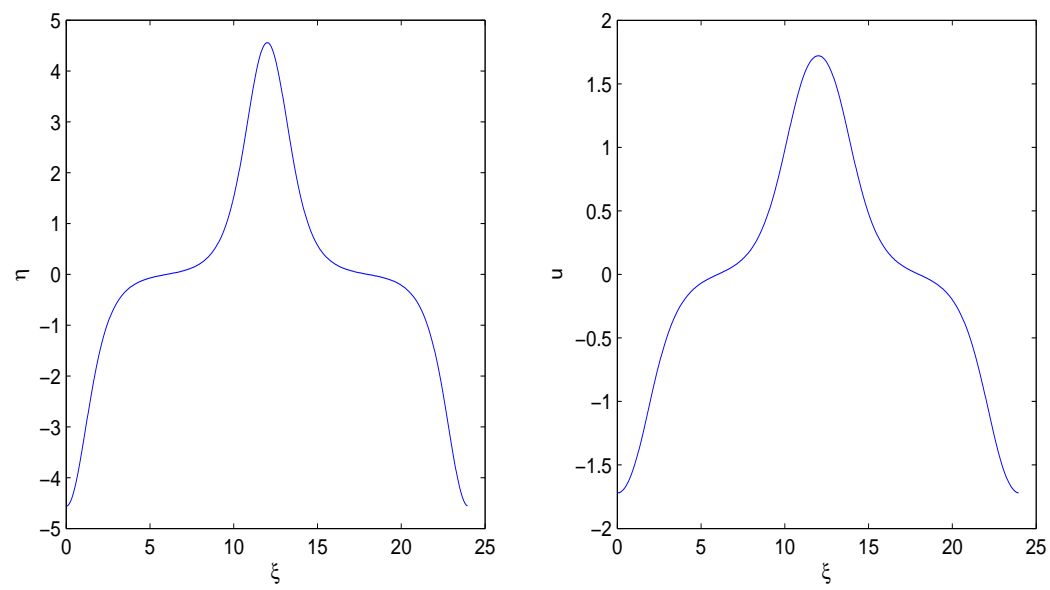

FIG. 6.10. Travelling-wave solution $(\eta, u)$ with half-period $l=12$, wave speed $c=2.5$, and $p=2$.

we obtain a system of $N+2$ nonlinear equations in the form

$$
F\left(\eta_{0}, \eta_{1}, \ldots, \eta_{N / 2}, u_{0}, u_{1}, \ldots, u_{N / 2}\right)=0,
$$

where the $N+2$ coefficients $\eta_{n}, u_{n}$ are the unknowns. System (6.4) is solved by Newton's iteration programmed in Matlab on a computer with core 2 duo processor running at $3.0 \mathrm{GHz}$ and $4 \mathrm{~Gb}$ Memory. Computation of the cosine series in (6.2) and the integrals in (6.3) is performed by using the FFT (Fast Fourier Transform) algorithm. The Jacobian of the vector field $F: \mathbb{R}^{N+2} \rightarrow \mathbb{R}^{N+2}$ is approximated by the second-order accurate formula

$$
J_{i, j} F(x) \approx \frac{F_{i}\left(x+h e_{j}\right)-F_{i}\left(x-h e_{j}\right)}{2 h}, \quad i, j=1, \ldots N+2,
$$



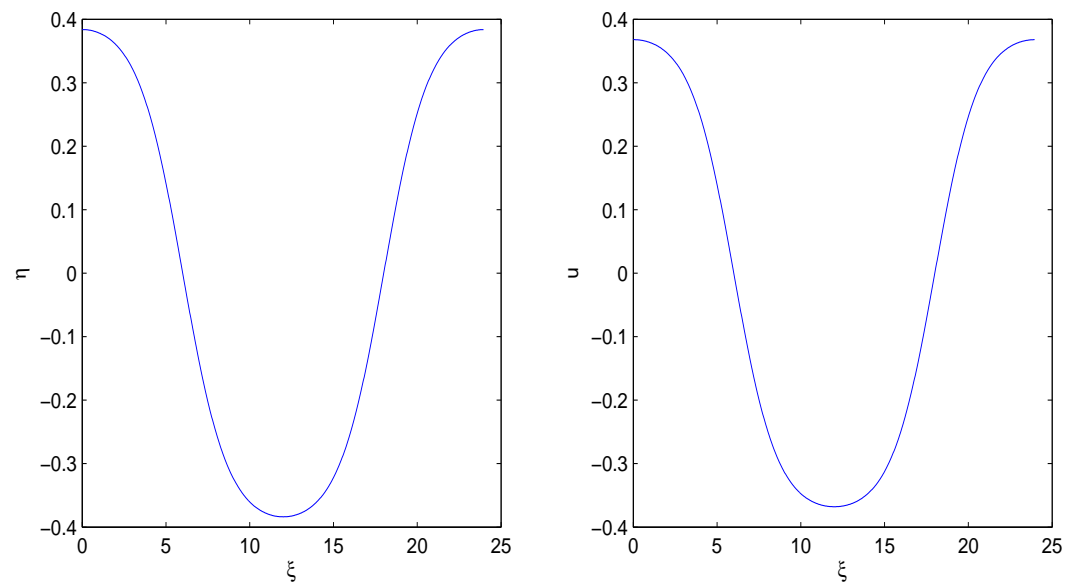

FIG. 6.11. Travelling-wave solution $(\eta, u)$ with half-period $l=12$, wave speed $c=1.1$, and $p=2$.
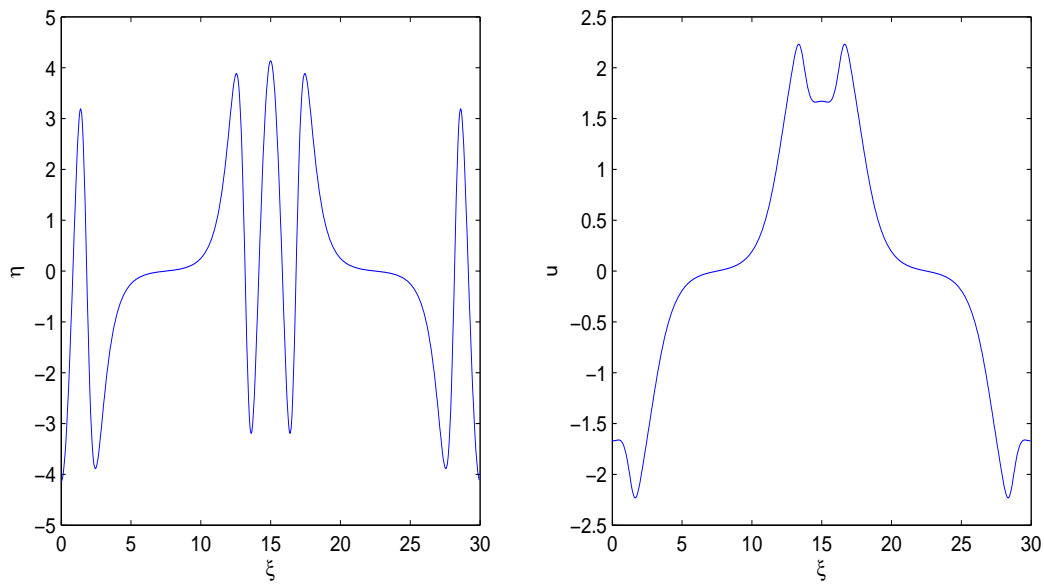

FIG. 6.12. Travelling-wave solution $(\eta, u)$ with half-period $l=15$, wave speed $c=3$, and $p=4$.

where $e_{j}=(0, \ldots 1, \ldots 0), h=0.001$. In all of the experiments presented in the next section we take $N=2^{9}$.

We point out that the numerical solver presented can be adapted to treat travelling-wave solutions of other well-known dispersive equations, such as the generalized KdV model [17], the Benjamin-Bona-Mahony equation [1], and the Benjamin-Ono equation [2]. Further work is in progress in adapting this scheme to consider solitarywave solutions (non-periodic) of the Boussinesq System (4.1) and of Benjamin-Ono systems [12], by using a basis of modified Chebyshev rational functions [15] more appropriate to manage problems in non-periodic spatial domains. These are topics under current research. 
6.1. Description of the numerical experiments. In this section the numerical scheme described in the previous section is employed to compute some travelling-wave solutions of System (4.1). In first place we set the wave velocity $c$ within the range given by condition (4.8).

Experiment 1: In first place we take $\delta=-0.5, \theta=-0.8, \mu=-0.1, c=2, p=1$, and half-period $l=10$. The initial point for Newton's iteration method is

$$
\eta_{0}(\xi)=\cos \left(\frac{\pi \xi}{l}\right), \quad u_{0}(\xi)=\cos \left(\frac{\pi \xi}{l}\right) .
$$

We stop Newton's iteration when the relative difference (henceforth called the method's tolerance) between two successive iterations is smaller than $1 e-14$. In this case, we obtain convergence in 41 iterations. The profiles obtained for $\eta$ and $u$ are presented in Figure 6.1. In order to verify the validity of the approximations computed, we use the spectral numerical solver introduced by the author in [24] which allows one to compute the time evolution of the solution of System (4.1) given initial profiles $\eta(x, 0), u(x, 0)$. This numerical solver was used in [24] for verifying some theoretical results in regard to System (4.1). It is expected that a travelling wave solution translates without changing its form for all time. An interesting observation which can be further verified with this numerical tool is that the fundamental period of the profile displayed in Figure 6.1 is really equal to 10 . We run the evolution solver on the spatial interval $[0,10]$, using as initial data the waveforms in Figure 6.1, $2^{8}$ FFT points on the spatial domain and $\Delta t=1 e-6$. The outcomes from this experiment are displayed in Figure 6.2 which shows the profiles of $\eta, u$ at $t=100$ superimposed with the initial data translated a distance of $100 c=200$ units to the right, since $c=2$ is the wave velocity in this case. We remark that in this experiment the travelling wave has propagated a distance of 20 times its fundamental period.

In Figure 6.3 we plot the absolute value of the difference between the profiles displayed in Figure 6.2. See that these profiles agree with a good accuracy $(1 e-8)$ showing that in fact the waveforms computed by Newton's iteration behave as periodic travelling-wave solutions of System (4.1). This verification was performed successfully for all experiments presented in this section and analogous results were obtained.

Observe that Newton's method is capturing a periodic travelling wave whose fundamental period is $1 / 2$ of the expected value 20 . We also observe this phenomenon in several simulations using different initial conditions. For instance, when

$$
\eta_{0}(\xi)=1+\cos \left(\frac{\pi \xi}{5}+\pi\right), \quad u_{0}(\xi)=1+\cos \left(\frac{\pi \xi}{5}+\pi\right),
$$

$l=5$, and the same parameters as in Experiment 1, Newton's iteration provides the approximation presented in Figure 6.4 in only 9 iterations with tolerance $1 e-14$. Observe that the fundamental period of the solution is 5 , which is $1 / 2$ the period of the initial conditions $\eta_{0}, u_{0}$.

Now we compute another travelling wave for different values of the modelling parameters $\delta=-0.5, \theta=-2, \mu=-1, c=1.5, p=1$, and $l=5$. The number of iterations required in this case is 7 . As a general rule, we observe that when the half-period $l$ increases, more Newton's iterations are required in order to achieve the tolerance imposed. The corresponding profiles for the travelling-wave solution computed $(\eta, u)$ are presented in Figure 6.5.

Experiment 2: In this set of experiments we increase the nonlinear exponent to $p=2$. The other parameters are $\delta=-0.5, \theta=-2, \mu=-1, c=1.5$, and $l=6$. In 
this case, the required tolerance is achieved in 8 Newton's iterations. The profiles of the travelling-wave solution are presented in Figure 6.6. The next experiment is performed for the model's parameters $\delta=-0.5, \theta=-0.8, \mu=-0.1, c=5, l=6$, and $p=2$. To avoid the case where Newton's method converges to the trivial solution $(\eta=0, u=0)$, we change the initial point to

$$
\eta_{0}(\xi)=3 \cos \left(\frac{\pi \xi}{l}\right), \quad u_{0}(\xi)=3 \cos \left(\frac{\pi \xi}{l}\right) .
$$

We obtain convergence with 6 iterations. The resulting travelling-wave solution $(\eta, u)$ is presented in Figure 6.7.

Experiment 3: In these experiments we consider larger values of the nonlinear exponent $p$. In Figure 6.8 we present the approximation obtained for the travellingwave solution corresponding to the model's parameters $\delta=-0.5, \theta=-0.8, \mu=-0.1$, $c=5, l=6$, and $p=3$. Tolerance is reached at 7 Newton's iterations.

On the other hand, in Figure 6.9 we plot the approximation to the travelling-wave solution for the same model's parameters except that we take $p=4$. In this numerical experiment, the initial point for Newton's method is taken as

$$
\eta_{0}(\xi)=1.5 \cos \left(\frac{\pi \xi}{l}\right), \quad u_{0}(\xi)=1.5 \cos \left(\frac{\pi \xi}{l}\right) .
$$

The number of Newton's iterations in this simulation is 12 .

Experiment 4: Finally we can use the numerical solver presented in order to explore existence of travelling-wave solutions of System (4.1) whose wave velocities do not satisfy condition (4.8).

In first place set $\delta=-0.5, \theta=-2, \mu=-1, l=12, c=2.5$, and $p=2$. For these parameters, observe that the range of velocity for which travelling-wave solutions exist according to the theory developed is the interval $[1.33,2]$. The result is presented in Figure 6.10. Other solutions are displayed in Figure 6.11 where $\delta=-0.5, \theta=-2, \mu=$ $-1, l=12, c=1.1$, and $p=2$, and in Figure 6.12 for the case $\delta=-0.5, \theta=-0.2, \mu=$ $-0.1, l=15, c=3$, and $p=4$. The initial conditions for Newton's iteration in these experiments are taken in the form

$$
\eta_{0}(\xi)=\cos \left(\frac{\pi \xi}{l}\right), \quad u_{0}(\xi)=\cos \left(\frac{\pi \xi}{l}\right) .
$$

The range of wave velocity given by condition (4.8) for the parameters used in the simulation in Figure 6.11 is [1.33, 2] and in Figure 6.12 is the interval [1,2].

Remark that in Experiments 1,2,3 we captured numerically a periodic travellingwave solution of the Boussinesq System (4.1) corresponding to each set of model's parameters and wave speed within the expected range, in perfect accordance with the mathematical theory presented in previous sections. Thus, our computer simulations illustrate and validate the theoretical results presented in this paper. In Experiment 4, this numerical tool also allowed us to discover new periodic travelling-wave solutions of System (4.1) which do not belong to the family of solutions proved to exist since their wave velocities are outside the interval given by condition (4.8). It remains as an open problem to extend the velocity range for which travelling-wave solutions of System (4.1) do exist.

A plausible question of great interest in pure and applied mathematical analysis is if the periodic solutions computed are orbitally unstable/stable with respect to small perturbations. This problem could also be investigated with the help of the Boussinesq 
solver introduced together with the numerical method developed by the author in [24]. Further research using this numerical Boussinesq solver is being conducted in order to discover novel geometrical properties of the periodic travelling-wave solutions computed and to determine whether they tend to a solitary-wave solution of System (1.1) when the wave's period $2 l$ goes to infinity, as observed in other dispersive-type systems such as KdV, Benjamin-Ono and Benney-Luke equations.

Acknowledgments. This work has been supported by the Universidad del Valle under the project No 7822. The author wishes to thank his lovely wife Norma Ortiz Cancino for her valuable help in preparing this manuscript.

\section{REFERENCES}

[1] J. Albert, On the decay of solutions of the generalized Benjamin-Bona-Mahony equation, J. Math. Anal. Appl., 141, 527-537, 1989.

[2] T.B. Benjamin, Internal waves of permanent form in fluids od great depth, J. Fluid. Mech., 29(3), 559-592, 1967.

[3] T.B. Benjamin, J.L. Bona, and D.K. Bose, Solitary-wave solutions of nonlinear problems, Phil. Trans. R. Soc. Lond. Ser A., 331, 195-244, 1990

[4] D.J. Benney and J.C. Luke, Interactions of permanent waves of finite amplitude, J. Math, Phys., 43, 309-313, 1964

[5] J.L. Bona, M. Chen, and J.C. Saut, Boussinesq equations and other systems for small-amplitude long waves in nonlinear dispersive media I: Derivation and the linear theory, J. Nonlin. Sci., 12, 283-318, 2002.

[6] J.L. Bona and M. Chen, A Boussinesq system for two-way propagation of nonlinear dispersive waves, Physica D., 116, 191-224, 1998.

[7] V. Bisognin, On the asymptotic behavior of the solutions of a nonlinear dispersive system of Benjamin-Bona-Mahony's type, Bollettino U.M.I., 7, 10-B, 99-128, 1996.

[8] M. Bjørkavåg and $\mathrm{H}$. Kalisch, Wave breaking in Boussinesq models for undular bores, Phys. Lett. A, 375(14), 1570-1578, 2011.

[9] M. Chen, Solitary-wave and multi-pulsed travelling-wave solutions of a Boussinesq system, Applicable Analysis, 75(1-2), 213-240, 2000.

[10] H. Chen, Existence of periodic travelling-wave solutions of nonlinear, dispersive wave equations, Nonlin., 17, 2041-2056, 2004.

[11] H. Chen, M. Chen, and N.V. Nguyen, Cnoidal wave solutions to Boussinesq systems, Nonlin., 20, 1443-1461, 2007.

[12] W. Choi and R. Camassa, Long internal waves of finite amplitude, Phys. Rev. Lett., 77(9), 1759-1762, 1996.

[13] A. Granas, The Leray-Schauder index and fixed point theory for arbitrary ANRs, Bull. Soc. Math. Fr., 100, 209-228, 1972.

[14] J.A. Gear and R. Grimshaw, Weak and strong interactions between internal solitary waves, Studies Appl. Math., 70, 235-258, 1984

[15] B.Y. Guo and Z.Q. Wang, Modified Chebyshev rational spectral method for the whole line, Proceedings of the Fourth International Conference on Dynamical Systems and Differential Equations, May, 24-27, 365-374, 2002.

[16] C.E. Kenig, G. Ponce, and L. Vega, On the generalized Korteweg- de Vries equation, Duke Math. J., 59(3), 585-610, 1989.

[17] D.J. Korteweg and G. de Vries, On the change of form of long waves advancing in a rectangular canal and on a new type of long stationary wave, Phil. Mag., 39, 422-443, 1985.

[18] M.A. Krasnosel'skii, Positive Solutions of Operator Equations, ed. LF Boron, Groningen: P. Noordhoff Ltd., 1964.

[19] M.A. Krasnosel'skii Topological Methods in the theory of nonlinear integral equations, ed. J Burlak, NY, USA: Pergamon Press, 1964.

[20] J. Morais, Global existence and decay of solutions of a coupled system of BBM-Burgers equations, Revista Matemtica Complutense, XIII, 2, 423-443, 2000.

[21] J.C. Muñoz Grajales and A. Nachbin, Dispersive wave attenuation due to orographic forcing, SIAM J. Appl. Math., 64(3), 977-1001, 2004.

[22] J.C. Muñoz Grajales and A. Nachbin, Stiff microscale forcing and solitary wave refocusing, Multiscale Model. Simul., 3, 680-705, 2006. 
[23] J.C. Muñoz Grajales and A. Nachbin, Improved Boussinesq-type equations for highly variable depth, IMA J. Appl. Math., 71, 600-633, 2006.

[24] J.C. Muñoz Grajales and I. Rivas, Existence and asymptotic decay for a Boussinesq-type model, J. Math. Anal. Appl., 345, 455-475, 2008.

[25] H.Y. Nguyen and F. Dias, A Boussinesq system for two-way propagation of interfacial waves, Phys. D., 237, 2365-2389, 2008. 